\title{
Regularity and structure of pullback attractors for reaction-diffusion type systems without uniqueness
}

\author{
Hongyong Cui ${ }^{\mathrm{a}, \mathrm{b}}$, José A. Langa ${ }^{\mathrm{b}}$, Yangrong $\mathrm{Li}^{\mathrm{a}}{ }^{\mathrm{a} *}$ \\ ${ }^{a}$ School of Mathematics and Statistics, Southwest University, Chongqing 400715, P.R. China. \\ ${ }^{b}$ Departamento de Ecuaciones Diferenciales Análisis Numérico, Universidad de Sevilla, Apdo. de Correos 1160, \\ 41080-Sevilla Spain
}

\begin{abstract}
In this paper, we study the pullback attractor for a general reaction-diffusion system for which the uniqueness of solutions is not assumed. We first establish some general results for a multi-valued dynamical system to have a bi-spatial pullback attractor, and then we find that the attractor can be backwards compact and composed of all the backwards bounded complete trajectories. As an application, a general reaction-diffusion system is proved to have an invariant $(H, V)$-pullback attractor $A=\{A(\tau)\}_{\tau \in \mathbb{R}}$. This attractor is composed of all the backwards compact complete trajectories of the system, pullback attracts bounded subsets of $H$ in the topology of $V$, and moreover

$$
\bigcup_{s \leqslant \tau} A(s) \quad \text { is precompact in } V, \quad \forall \tau \in \mathbb{R} .
$$

A non-autonomous Fitz-Hugh-Nagumo equation is studied as a specific example of the reactiondiffusion system.
\end{abstract}

\section{Contents}

1 Introduction $\quad 2$

2 Backwards bounded pullback attractors for m-NDS $\quad 3$

2.1 Existence of a backwards bounded pullback attractor . . . . . . . . . . . . 4

2.2 Invariance of pullback attractors for strict m-NDS $\ldots \ldots \ldots \ldots \ldots \ldots$

2.3 Structure of pullback attractors for strict m-NDS $\ldots \ldots \ldots \ldots \ldots$

$\begin{array}{lll}3 & \text { Regularity of pullback attractors for m-NDS } & 11\end{array}$

3.1 Bi-spatial pullback attractors for strongly dispersive m-NDS . . . . . . . . . . . . 11

3.2 Backwards precompactness of pullback attractors for m-NDS . . . . . . . . . . . 13

4 Applications to a reaction-diffusion type system without uniqueness $\quad 14$

4.1 Preliminary settings . . . . . . . . . . . . . . . . . . 14

4.2 Backwards precompact attractors in $H \ldots \ldots \ldots \ldots \ldots \ldots \ldots \ldots$

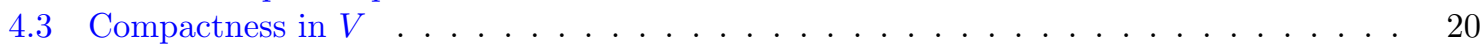

4.4 An example: attractors for non-autonomous Fitz-Hugh-Nagumo equations . . . . . . . 26 $\mathrm{Li})$

* Corresponding author

Email addresses: h.cui@outlook.com (Hongyong Cui), langa@us.es (José A. Langa), liyr@swu.edu.cn (Yangrong 


\section{Introduction}

A multi-valued non-autonomous dynamical system (m-NDS) is generated by an evolution equation whose solution is unnecessarily unique. The study on m-NDS has particular significance since m-NDS are often established under weaker conditions than usual (single-valued) dynamical systems. For an m-NDS $\phi$ on a Banach space $X$, a pullback attractor $A=\{A(\tau)\}_{\tau \in \mathbb{R}}$ is defined as a compact and negatively invariant non-autonomous set which attracts each bounded subset of $X$ (see Definition 2.7). This can be regarded as a generalization of pullback attractors for single-valued dynamical systems which have been studied widely, see for instance $[3,4, \underline{5,} \underline{11]}$.

In this paper, we study the structure and regularity of the pullback attractor for the m-NDS generated by the following reaction-diffusion equation defined on a bounded domain $\mathcal{O} \subset \mathbb{R}^{N}$ :

$$
\left\{\begin{array}{l}
\frac{\mathrm{d} u}{\mathrm{~d} t}-a \triangle u+f(t, u)=g(x, t), \\
\left.u\right|_{\partial \mathcal{O}}=0 \\
\left.u\right|_{t=\tau}=u_{0},
\end{array}\right.
$$

where $(t, x) \in(\tau, \infty) \times \mathcal{O}, u=\left(u^{1}(t, x), \cdots, u^{d}(t, x)\right):(\tau, \infty) \times \mathcal{O} \rightarrow \mathbb{R}^{d}, a$ is a real $d \times d$ matrix with a positive symmetric part $\frac{a+a^{t}}{2} \geqslant \beta I$ for some $\beta>0$, and $f(t, u) \in C\left((\tau, \infty) \times \mathbb{R}^{d} ; \mathbb{R}^{d}\right)$ satisfies some conditions unable to ensure the uniqueness of solutions (see Section 4). The most direct example of this system is the classical reaction-diffusion equation (taking $d=1$ ) frequently seen in the literature. Nevertheless, the system (1.1) covers more models, such as the Fitz-Hugh-Nagumo equations

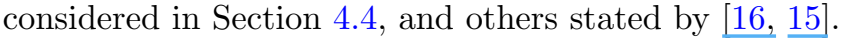

We get three aims in this work. The first is the existence of the $(H, V)$-pullback attractor $A=$ $\{A(\tau)\}_{\tau \in \mathbb{R}}$ for (1.1), which is a non-autonomous set pullback attracting bounded sets of $H$ under the topology of $V$, where

$$
H:=\left(L^{2}(\mathcal{O})\right)^{d}, \quad V:=\left(H_{0}^{1}(\mathcal{O})\right)^{d} .
$$

This is a study of bi-spatial attractors which attracted much attention these years due to their higher regularity and stronger attracting ability compared with usual attractor, see $[7,13,12]$ for single-valued non-autonomous/random cocycles and [21, 19] for multi-valued semi-groups and random cocycles, respectively. In this paper, we develop a study for m-NDS which can be regarded as an extension of the bi-spacial attractor theory on one hand, and is interesting because of the multi-valued feature on the other hand.

The second aim is to establish the backwards precompactness of $A$, that is,

$$
\bigcup_{s \leqslant \tau} A(s) \text { is precompact in } V, \quad \forall \tau \in \mathbb{R} .
$$

This subject is new since the compactness of a pullback attractor is often considered for each fixed "section" $A(\tau)$ in the literature. To achieve this, we first establish some sufficient conditions to ensure an attractor to be backwards precompact, and then apply them to the system (1.1) when the external force $g$ is backwards translation bounded, namely, $g \in L_{\text {loc }}^{2}(\mathbb{R} ; H)$ with

$$
\sup _{\tau<0} \int_{\tau-1}^{\tau}\|g(s)\|^{2} \mathrm{~d} s<\infty .
$$

This condition is shown weaker than translation bounded condition considered in [8] and stronger than tempered conditions seen in recent studies for random dynamical systems, such as [17] and references therein. Establishing a non-autonomous set $\{K(\tau)\}_{\tau \in \mathbb{R}}$ which is absorbing and increasing in $\tau$ plays a key role to obtain the backwards compactness of the attractor.

The third main aim is to characterize the pullback attractor $A$ by complete trajectories. For strict m-NDS we first introduce the concept of an m-NDS generating smooth trajectories, and then prove 
that backwards bounded pullback attractors for such systems are composed of backwards bounded trajectories, see Theorem 2.24. Note that, the class of dynamical systems who generate smooth trajectories is rather general. A direct example is the so-called generalized dynamical systems, first studied by Ball $[1]$ and recently studied by Simsen [14] for generalized semiflows and Kapustyan et al.

[9] for generalized processes, etc. Therefore, Theorem 2.24 is interesting even in single-valued cases as single-valued systems must be strict.

We carry out this work as follows.

In Section 2, we first make some necessary definitions, and then establish some abstract results on the existence of a backwards bounded pullback attractor and on the structure of pullback attractors is also studied in this section.

In Section 3, we first establish a result on the existence of a bi-spatial pullback attractor by making a direct comparison on bi-spatial and single-spatial attractors, see Theorem 3.9. This result indicates an interesting fact that the regularity of a pullback attractor, once the attractor exists, is determined by the dynamical compactness of the system. Then we establish several sufficient conditions to verify the backwards precompactness of a pullback attractor. These conditions are established by strengthen the dynamical compactness of the system (see Theorem 3.18), and this coincides with the observation that the regularity properties of the attractor have a close relationship with the dynamical compactness.

In Section 4, we study the system (1.1) and find that, under certain conditions the system (1.1) admits an $(H, V)$-pullback attractor which is invariant and composed of backwards compact complete trajectories. A Fitz-Hugh-Nagumo equation is studied as a specific example.

\section{Backwards bounded pullback attractors for m-NDS}

Throughout this paper, let $\left(X,\|\cdot\|_{X}\right)$ be a separable Banach space. Denote by $C(X)$ the set of all nonempty closed subsets of $X$ and $\mathcal{B}(X) \subset C(X)$ the set of all bounded closed subsets of $X$. $\mathbb{R}^{+}=[0, \infty)$. The Hausdorff semi-distance between two nonempty subsets $A$ and $B$ of $X$ is defined by $\operatorname{dist}_{X}(A, B):=\sup _{a \in A} \inf _{b \in B}\|a-b\|_{X}$. Denote the open $\delta$-neighborhood of a subset $B$ of $X$ by $\mathcal{N}_{\delta}(B)$. Suppose $\left\{\theta_{t}\right\}_{t \in \mathbb{R}}$ is a group of translation operators acting on $\mathbb{R}$ defined by

$$
\theta_{s} \tau=\tau+s, \quad \forall s, \tau \in \mathbb{R} .
$$

Definition 2.1. A non-autonomous set $D=\{D(\tau)\}_{\tau \in \mathbb{R}}$ in $X$ is a mapping: $\mathbb{R} \rightarrow C(X), \tau \mapsto D(\tau)$. The collection of all the non-autonomous sets in $X$ is denoted by $\mathcal{D}_{X}$.

Definition 2.2. A non-autonomous set $D$ is called compact/bounded (in $X)$ if, for every $\tau \in \mathbb{R}, D(\tau)$ is compact/bounded (in $X$ ). $D$ is called backwards precompact/bounded (in $X$ ) if

$$
\bigcup_{s \leqslant \tau} D(s) \text { is precompact/bounded (in } X \text { ) for every } \tau \in \mathbb{R} \text {. }
$$

Definition 2.3. A multi-valued mapping $\phi: \mathbb{R}^{+} \times \mathbb{R} \times X \rightarrow C(X)$ is called an m-NDS on $X$ if

(1) $\phi(0, \tau, \cdot)$ is the identity operator on $X, \forall \tau \in \mathbb{R}$;

(2) $\phi(t+s, \tau, \cdot) \subset \phi(t, \tau+s, \phi(s, \tau, \cdot)), \forall t, s \in \mathbb{R}^{+}, \tau \in \mathbb{R}$.

Moreover, if $\phi(t+s, \tau, \cdot)=\phi\left(t, \theta_{s} \tau, \phi(s, \tau, \cdot)\right)$, then the m-NDS $\phi$ is called a strict m-NDS.

Definition 2.4. An m-NDS $\phi$ is called upper semi-continuous at $x_{0}$ if for every $(t, \tau) \in \mathbb{R}^{+} \times \mathbb{R}$ and every neighborhood $\mathcal{U}$ of the set $\phi\left(t, \tau, x_{0}\right)$ there exists a $\delta>0$ such that

$$
\phi(t, \tau, x) \subset \mathcal{U} \text { for all } x \in \mathcal{N}_{\delta}\left(x_{0}\right) .
$$

$\phi$ is called upper semi-continuous in $X$ if it is upper semi-continuous everywhere in $X$. 
Remark 2.5. [2] Note that if a mapping $\phi(t, \tau, \cdot)$ is upper semi-continuous at $x_{0}$, then for all $\epsilon>0$ there exists $\delta(\epsilon)>0$ such that

$$
\operatorname{dist}_{X}\left(\phi(t, \tau, y), \phi\left(t, \tau, x_{0}\right)\right)<\epsilon \text { for any } y \in \mathcal{N}_{\delta}\left(x_{0}\right) .
$$

The converse is true when each value of $\phi$ is compact.

Definition 2.6. (1) We say $K \in \mathcal{D}_{X}$ is pullback attracting if for every $D \in \mathcal{B}(X)$ we have

$$
\lim _{t \rightarrow \infty} \operatorname{dist}_{X}\left(\phi\left(t, \theta_{-t} \tau, D\right), K(\tau)\right)=0, \quad \text { for every } \tau \in \mathbb{R} .
$$

(2) A mapping $B \in \mathcal{D}_{X}$ is called a pullback absorbing set if for every $D \in \mathcal{B}(X)$ there exists a $T=T(\tau, D)>0$ such that

$$
\phi\left(t, \theta_{-t} \tau, D\right) \subset B(\tau), \quad \text { for all } t \geqslant T .
$$

Definition 2.7. A mapping $A \in \mathcal{D}_{X}$ is called a pullback attractor in $X$ for the m-NDS $\phi$ if

1. $A$ is compact in $X$;

2. $A$ is pullback attracting;

3. $A$ is negatively invariant, namely, $A\left(\theta_{t} \tau\right) \subset \phi(t, \tau, A(\tau)), \forall t \in \mathbb{R}^{+}, \tau \in \mathbb{R}$.

Remark 2.8. Clearly, backwards bounded pullback attractor must be unique.

Remark 2.9. It is interesting to observe that, most generally, a pullback attractor $A$ is not always contained in any pullback absorbing set $B$ in the sense that $A(\tau) \subset B(\tau), \forall \tau \in \mathbb{R}$. But the inclusion must hold if $A$ is backwards bounded in $X$. This can be seen from the relation

$$
A(\tau) \subset \phi\left(t, \theta_{-t} \tau, A\left(\theta_{-t} \tau\right)\right) \subset \phi\left(t, \theta_{-t} \tau, D_{\tau}\right) \subset B(\tau), \text { for all } t \text { large enough. }
$$

\subsection{Existence of a backwards bounded pullback attractor}

As for single-valued dynamical systems, the main object to study the attractor is the pullback $\omega$-limit set for the m-NDS $\phi$. For some $D \in \mathcal{B}(X)$, the $\omega$-limit set of $D$ is a mapping $\omega(D, \cdot): \mathbb{R} \rightarrow 2^{X}$ defined by

$$
\omega(D, \tau)=\bigcap_{s>0}{\overline{\bigcup_{t>s} \phi\left(t, \theta_{-t} \tau, D\right)}}^{X}, \quad \forall \tau \in \mathbb{R},
$$

where the closure is taken under the norm topology of $X$. Note that $y \in \omega(D, \tau)$ iff there exist $t_{n} \rightarrow \infty$ and $y_{n} \in \phi\left(t_{n}, \theta_{-t_{n}} \tau, D\right)$ such that $y_{n} \stackrel{X}{\rightarrow} y$. Moreover, we have the following lemma.

Definition 2.10. Let $D \in \mathcal{B}(X)$. Then the m-NDS $\phi$ is called $D$-pullback asymptotically compact if for every sequence $t_{n} \rightarrow \infty$ and $\tau \in \mathbb{R}$, any sequence $\left\{y_{n}\right\}$ with $y_{n} \in \phi\left(t_{n}, \theta_{-t_{n}} \tau, D\right)$ is precompact in $X$. $\phi$ is called pullback asymptotically compact if it is $D$-asymptotically compact for every $D \in \mathcal{B}(X)$.

Remark 2.11. Such a dynamical compactness property is also termed as pullback asymptotically upper semi-compact in the literature, see [21, 18].

Lemma 2.12. Suppose $\phi$ is an $m$-NDS upper semi-continuous in $X$. Let $\phi$ be D-pullback asymptotically compact for some $D \in \mathcal{B}(X)$. Then,

(1) for all $\tau \in \mathbb{R}, \omega(D, \tau)$ is non-empty and compact in $X$;

(2) $\omega(D, \cdot)$ pullback attracts $D$, that is,

$$
\lim _{t \rightarrow \infty} \operatorname{dist}_{X}\left(\phi\left(t, \theta_{-t} \tau, D\right), \omega(D, \tau)\right)=0, \quad \forall t \in \mathbb{R}
$$


(3) $\omega(D, \cdot)$ is negatively invariant, i.e. $\omega\left(D, \theta_{t} \tau\right) \subset \phi(t, \tau, \omega(D, \tau))$ for all $t \geqslant 0$.

Proof. First observe that $y \in \omega(D, \tau)$ iff there exist $t_{n} \rightarrow \infty$ and $y_{n} \in \phi\left(t_{n}, \theta_{-t_{n}} \tau, D\right)$ such that $y_{n} \stackrel{X}{\rightarrow} y$.

(1) To see $\omega(D, \tau)$ is non-empty, we take a sequence $y_{n} \in \phi\left(t_{n}, \theta_{-t_{n}} \tau, D\right)$ with $t_{n} \rightarrow \infty$. By the $D$-pullback asymptotic compactness of $\phi$ we can find a $y \in X$ such that, up to a subsequence, $y_{n} \stackrel{X}{\rightarrow} y$. Therefore, $y \in \omega(B, \tau)$ and $\omega(B, \tau)$ is non-empty.

We now prove the $Y$-compactness. Given a sequence $\left\{y_{n}\right\} \subset \omega(D, \tau)$, we can find $t_{n} \rightarrow \infty$ and $z_{n} \in \phi\left(t_{n}, \theta_{-t_{n}} \tau, D\right)$ such that $\left\|y_{n}-z_{n}\right\|_{X}<1 / n$. By the $D$-asymptotic compactness of $\phi$ again we know $z_{n_{k}} \stackrel{X}{\rightarrow} z$ for some $z \in \omega(D, \tau)$. Thus, $y_{n_{k}} \stackrel{X}{\rightarrow} z$ and then $\omega(D, \tau)$ is compact in $X$.

(2) We prove the pullback $D$-attracting property by contradiction. Suppose it does not hold, then there exist an $\epsilon>0$ and $y_{n} \in \phi\left(t_{n}, \theta_{-t_{n}} \tau, D\right)$ with $t_{n} \rightarrow \infty$ such that

$$
\operatorname{dist}_{X}\left(y_{n}, \omega(D, \tau)\right)>\epsilon, \quad \forall n \in \mathbb{N} .
$$

But from the $D$-pullback asymptotic compactness of $\phi$ it follows that $y_{n_{k}} \stackrel{X}{\rightarrow} y$ for some $y \in \omega(B, \tau)$. Here we arrive at a contradiction.

(3) We now prove the negative invariance. For any $y \in \omega\left(D, \theta_{t} \tau\right)$, there exists a sequence $y_{n} \in$ $\phi\left(t_{n}, \theta_{-t_{n}} \theta_{t} \tau, x_{n}\right)$ with $x_{n} \in D$ and $t_{n} \rightarrow \infty$ such that $y_{n} \stackrel{X}{\rightarrow} y$. Notice that

$$
\phi\left(t_{n}, \theta_{-t_{n}} \theta_{t} \tau, x_{n}\right) \subset \phi\left(t, \tau, \phi\left(t_{n}-t, \theta_{t-t_{n}} \tau, x_{n}\right)\right), \quad \forall t_{n}>t,
$$

thus $y_{n} \in \phi\left(t, \tau, z_{n}\right)$ for some $z_{n} \in \phi\left(t_{n}-t, \theta_{t-t_{n}} \tau, x_{n}\right)$. By the pullback $D$-asymptotic compactness again we see that $z_{n_{k}} \stackrel{X}{\longrightarrow} z \in \omega(D, \tau)$. Therefore, since $x \mapsto \phi(t, \tau, x)$ is upper semi-continuous in $X$ with closed values, we have

$$
y \in \phi(t, \tau, z) \subset \phi(t, \tau, \omega(D, \tau)),
$$

which completes the proof.

Remark 2.13. Slightly modifying the proof one can establish the previous lemma also for m-NDS not upper semi-continuous but with closed graph, see [6] and also [7].

Theorem 2.14. Let $\phi$ be an $m-N D S$ on $X$. If

(i) $\phi$ is asymptotically compact;

(ii) $\phi$ has a bounded absorbing set $B \in \mathcal{D}_{X}$;

(iii) $\phi$ is upper semi-continuous in $X$,

then the non-autonomous mapping $A=\{A(\tau)\}_{\tau \in \mathbb{R}} \in \mathcal{D}_{X}$ defined by

$$
A(\tau)=\bigcup_{D \in \mathcal{B}(X)} \omega(D, \tau), \quad \forall \tau \in \mathbb{R}
$$

has properties

1. A is nonempty and bounded in $X$;

2. A is pullback attracting;

3. A is negatively invariant. 
Proof. Properties 2 and 3 and the non-empty property follow from Lemma 2.12. We now prove the boundedness of $A(\tau)$ for $\tau \in \mathbb{R}$ arbitrarily fixed. Since $B \in \mathcal{D}_{X}$ is a bounded absorbing set, for any $D \in \mathcal{B}(X)$ there is a time $T>0$ such that

$$
\bigcup_{t \geqslant T} \phi\left(t, \theta_{-t} \tau, D\right) \subset B(\tau)
$$

from which and the closedness of $B(\tau)$ it follows that

$$
\omega(D, \tau)=\bigcap_{s>0} \overline{\bigcup_{t>s} \phi\left(t, \theta_{-t} \tau, D\right)} \subset \overline{\bigcup_{t \geqslant T} \phi\left(t, \theta_{-t} \tau, D\right)} \subset B(\tau) .
$$

Thus $A(\tau) \subset B(\tau)$ is bounded in $X$.

Note that the non-autonomous set $A$ defined by Theorem 2.14 is not compact in general. To prove the compactness of $A$ we need to make assumptions on the pullback absorbing set. More precisely, we have the following result.

Theorem 2.15. Suppose conditions of Theorem 2.14 hold. Moreover, we suppose the absorbing set $B$ is such that, for every $\tau \in \mathbb{R}$ and $D \in \mathcal{B}(X), B(\tau)$ pullback absorbs $D$ at every $s \leqslant \tau$, that is,

$$
\text { for every } s \leqslant \tau \text { there is a time } T=T(D, s) \text { such that } \bigcup_{t \geqslant T} \phi\left(t, \theta_{-t} s, D\right) \subset B(\tau) .
$$

Then $A \in \mathcal{D}_{X}$ defined by (2.1) is the pullback attractor for $\phi$. Moreover, $A$ is backwards bounded, and

$$
A(\tau)=\omega(B(\tau), \tau), \quad \forall \tau \in \mathbb{R} .
$$

Proof. To prove $A$ is the pullback attractor, by Theorem 2.14 it suffices to show the compactness of $A$. For every $D \in \mathcal{B}(X)$, by $(2.2)$ we have

$$
\omega(D, s) \subset \overline{\bigcup_{t \geqslant T} \phi\left(t, \theta_{-t} s, D\right)} \subset B(\tau), \quad \forall s \leqslant \tau,
$$

which along with the negative invariance of $\omega(D, \tau)$ implies that

$$
\begin{aligned}
\omega(D, \tau) & \subset \phi\left(\tau-s, \theta_{-(\tau-s)} \tau, \omega(D, s)\right) \\
& \subset \phi\left(\tau-s, \theta_{-(\tau-s)} \tau, B(\tau)\right) \text { for all } s \leqslant \tau .
\end{aligned}
$$

Therefore,

$$
\omega(D, \tau) \subset \bigcap_{s \leqslant \tau} \overline{\bigcup_{t \geqslant \tau-s} \phi\left(t, \theta_{-t} \tau, B(\tau)\right)}=\omega(B(\tau), \tau), \quad \forall D \in \mathcal{B}(X),
$$

and thereby

$$
A(\tau) \subset \omega(B(\tau), \tau)
$$

is precompact in $X$. On the other hand, since $B(\tau) \in \mathcal{B}(X)$ and $\omega(B(\tau), \tau) \subset A(\tau)$, thereby

$$
A(\tau)=\omega(B(\tau), \tau)
$$

is closed and thereby compact in $X$. In addition, (2.3) implies

$$
\bigcup_{s \leqslant \tau} A(s) \subset B(\tau)
$$

and thereby the backwards boundedness is also clear. 
On Theorem 2.15 we make following remarks.

Remark 2.16. If the pullback absorbing set $B=\{B(\tau)\}_{\tau \in \mathbb{R}} \in \mathcal{D}_{X}$ is increasing in $\tau$, that is,

$$
B(s) \subseteq B(\tau) \text { whenever } s \leqslant \tau
$$

then the condition (2.2) is fulfilled. Moreover, it is possible to establish a pullback absorbing set $B$ satisfying (2.2) with $T$ independent of $s$ and $\tau$ in specific applications, and in this case the condition (2.2) is equivalent to that there is an increasing bounded absorbing set $B^{\prime}$ for $\phi$. Indeed, if $B$ is a bounded absorbing set with (2.2), then the non-autonomous set $B^{\prime}$ defined by

$$
B^{\prime}(\tau)=\bigcap_{s \geqslant \tau} B(s), \quad \forall \tau \in \mathbb{R}
$$

is readily checked an increasing bounded absorbing set.

Remark 2.17. It is interesting to notice that if $B$ is moreover compact, then (2.4) implies $A$ is backwards precompact. We will discuss the backwards compactness later in Section 3.

\subsection{Invariance of pullback attractors for strict $m-N D S$}

In this part and motivated by Wang \& Zhou $\underline{[20]}$ where uniform attractors for m-NDS were studied, we prove the invariance of a pullback attractor for strict m-NDS.

Theorem 2.18. The backwards bounded pullback attractor $A$ of a strict $m$-NDS $\phi$ must be invariant.

Proof. Clearly, the negative invariance of $A$ holds by definition. Now by two steps we prove the positive invariance, i.e.,

$$
\phi(t-\tau, \tau, A(\tau)) \subset A(t), \quad \forall t \geqslant \tau .
$$

Step 1, we prove the non-autonomous set $Q=\{Q(\tau)\}_{\tau \in \mathbb{R}}$ is invariant, where

$$
Q(\tau)=\bigcup_{s \leqslant \tau} \phi(\tau-s, s, A(s)) .
$$

Let $t \geqslant \tau$. First notice that, by the strictness,

$$
\begin{aligned}
\phi(t-\tau, \tau, Q(\tau)) & =\phi\left(t-\tau, \tau, \bigcup_{s \leqslant \tau} \phi(\tau-s, s, A(s))\right) \\
& =\bigcup_{s \leqslant \tau} \phi(t-\tau, \tau, \phi(\tau-s, s, A(s))) \\
& =\bigcup_{s \leqslant \tau} \phi(t-s, s, A(s)) \subset Q(t),
\end{aligned}
$$

and thus the positive invariance of $Q$ is clear. Now we prove the negative invariance. Note that for every $y \in Q(t)$ there exist an $s \leqslant t$ and $x \in A(s)$ such that $y \in \phi(t-s, s, x)$. Then two cases should be taken into account.

Case $1, s \geqslant \tau$. Since by the negative invariance of $A$ there is an $x^{\prime} \in A(\tau)$ such that $x \in$ $\phi\left(s-\tau, \tau, x^{\prime}\right)$,

$$
\begin{aligned}
y \in \phi(t-s, s, x) & \subset \phi\left(t-s, s, \phi\left(s-\tau, \tau, x^{\prime}\right)\right)=\phi\left(t-\tau, \tau, x^{\prime}\right) \\
& \subset \phi(t-\tau, \tau, A(\tau)) \subset \phi(t-\tau, \tau, Q(\tau)),
\end{aligned}
$$

which implies $Q(t) \subset \phi(t-\tau, \tau, Q(\tau))$ in this case. 
Case 2, $s<\tau$. Then by the negative invariance of $A$ and for some $r<s$,

$$
\begin{aligned}
y \in \phi(t-s, s, A(s)) & =\phi(t-\tau, \tau, \phi(\tau-s, s, A(s))) \\
& \subset \phi(t-\tau, \tau, \phi(\tau-s, s, \phi(s-r, r, A(r))) \\
& =\phi(t-\tau, \tau, \phi(\tau-r, r, A(r))),
\end{aligned}
$$

which implies $Q(t) \subset \phi(t-\tau, \tau, Q(\tau))$ and thereby $Q$ is negatively invariant. Step 1 is concluded.

Step 2 , let us prove (2.5). Recall that by the backwards boundedness of $A$, there is a $D_{\tau} \in \mathcal{B}(X)$ such that

$$
\bigcup_{s \leqslant \tau} A(s) \subset D_{\tau}
$$

Then since $Q$ is invariant, for all $t \geqslant \tau \geqslant s$ we have

$$
\begin{aligned}
\phi(t-\tau, \tau, A(\tau)) & \subset Q(t)=\phi(t-s, s, Q(s))=\bigcup_{\gamma \leqslant s} \phi(t-s, s, \phi(s-\gamma, \gamma, A(\gamma))) \\
& \subset \bigcup_{\gamma \leqslant s} \phi\left(t-s, s, \phi\left(s-\gamma, \gamma, D_{\tau}\right)\right)=\bigcup_{\gamma \leqslant s} \phi\left(t-\gamma, \gamma, D_{\tau}\right) \\
& =\bigcup_{t^{\prime} \geqslant t-s} \phi\left(t^{\prime}, \theta_{-t^{\prime}} t, D_{\tau}\right) \quad\left(\text { let } t^{\prime}=t-\gamma\right),
\end{aligned}
$$

which implies

$$
\phi(t-\tau, \tau, A(\tau)) \subset \bigcap_{s \leqslant \tau} \bigcup_{t^{\prime} \geqslant t-s} \phi\left(t^{\prime}, \theta_{-t^{\prime}} t, D_{\tau}\right) \subset \omega\left(D_{\tau}, t\right) \subset A(t), \quad \forall t \geqslant \tau .
$$

Therefore, (2.5) holds and the proof is complete.

Remark 2.19. This result was also established by Coti Zelati and Kalita [6, Proposition 4.3] under the assumption that

$$
\text { there exists } t_{\star}>0 \text { such that } A(t) \subset \phi\left(t_{\star}, t-t_{\star}, A\left(t-t_{\star}\right)\right), \forall t \in \mathbb{R} .
$$

The backwards boundedness of the attractor plays a key role in the proof of the invariance. For the pullback attractor $A=\{A(\tau)\}_{\tau \in \mathbb{R}}$ defined by (2.1), we have the following observation when $A$ has no backwards boundedness. This observation indicates an interesting fact that, though the invariance of the pullback attractor remains unknown, any trajectories from a "section" $A(s)$ cannot entirely bypass any section behind.

Proposition 2.20. Suppose $A=\{A(\tau)\}_{\tau \in \mathbb{R}}$ defined by (2.1) is a pullback attractor, unnecessarily backwards bounded, of a strict and upper semi-continuous $m-N D S \phi$. Then for any $t \geqslant s$,

$$
\phi(t-s, s, x) \cap A(t) \neq \emptyset, \quad \forall x \in A(s) .
$$

Proof. We prove by contradiction. Suppose the proposition is not true for some $x \in A(s)$ and $s<t$, then by the closedness of $\phi(t-s, s, x)$ and $A(t)$ we can find a $\delta>0$ such that

$$
\inf _{b \in \phi(t-s, s, x)} \operatorname{dist}_{X}(b, A(t))>\delta .
$$

Since, by (2.1), there is a $D \in \mathcal{B}(X)$ such that

$$
x \in \omega(D, s),
$$


there exist sequences $t_{n} \rightarrow \infty$ and $y_{n} \in \phi\left(t_{n}, s-t_{n}, D\right)$ such that

$$
y_{n} \rightarrow x
$$

Thus by the upper semi-continuity of $\phi$ we find that

$$
\operatorname{dist}_{X}\left(\phi\left(t-s, s, y_{n}\right), \phi(t-s, s, x)\right)<\delta / 4 \quad \text { for } n \text { large enough, }
$$

which implies the existence of a $b \in \phi(t-s, s, x)$ and a $c \in \phi\left(t-s, s, y_{n}\right)$ such that

$$
\|b-c\|_{X}<\delta / 2 \text {. }
$$

On the other hand, since $A(t)$ pullback attracts $D$ at time $t$ and by the strictness of $\phi$, we have

$$
\begin{aligned}
\operatorname{dist}_{X}(c, A(t)) & \leqslant \operatorname{dist}_{X}\left(\phi\left(t-s, s, y_{n}\right), A(t)\right) \\
& \leqslant \operatorname{dist}_{X}\left(\phi\left(t-s, s, \phi\left(t_{n}, s-t_{n}, D\right)\right), A(t)\right) \\
& =\operatorname{dist}_{X}\left(\phi\left(t-\left(s-t_{n}\right), s-t_{n}, D\right), A(t)\right) \\
& <\delta / 2 \quad(\text { for all large } n) .
\end{aligned}
$$

Therefore, from (2.6) and (2.7) it follows

$$
\operatorname{dist}_{X}(b, A(t))<\delta
$$

where $b \in \phi(t-s, s, x)$; a contradiction.

\subsection{Structure of pullback attractors for strict $m-N D S$}

In this part, we characterize pullback attractors of strict m-NDS by complete trajectories.

Definition 2.21. A complete trajectory $\xi$ of an m-NDS $\phi$ on $X$ is defined as a mapping in $C(\mathbb{R} ; X)$ which enjoys the property

$$
\xi(t) \in \phi(t-s, s, \xi(s)), \quad \forall t \geqslant s .
$$

If $\xi$ is moreover backwards bounded, then $\xi$ is called a backwards bounded complete trajectory.

It is straightforward to obtain the following lemma.

Lemma 2.22. If the pullback attractor $A$ exists, then every backwards bounded complete trajectory $\xi$ must be contained in A, that is,

$$
\xi(\tau) \in A(\tau), \forall \tau \in \mathbb{R}
$$

Definition 2.23. We say an m-NDS $\phi$ generates smooth trajectories, if for each $x \in X$ and $\tau \in \mathbb{R}$ there exists a family

$$
\mathcal{R}_{x}^{\tau}:=\{r \in C([\tau, \infty) ; X): r(t) \in \phi(t-s, s, r(s)) \text { and } r(\tau)=x, \forall t \geqslant s \geqslant \tau\}
$$

such that

$$
\phi(t, \tau, x)=\bigcup_{r \in \mathcal{R}_{x}^{\tau}} r(\tau+t), \quad \forall t \geqslant 0 .
$$

The following result provides a sufficient condition under which the backwards bounded pullback attractor of a strict m-NDS is composed of backwards bounded complete trajectories. 
Theorem 2.24. Suppose $\phi$ is a strict $m$-NDS on Banach space $X$ with a backwards bounded pullback attractor $A$. If $\phi$ generates smooth trajectories, then

$$
A(\tau)=\{\xi(\tau): \xi \text { is a backwards bounded complete trajectory of } \phi\}, \forall \tau \in \mathbb{R} .
$$

Proof. By Lemma 2.22 it suffices to prove the $\subset$ relation. Let $z \in A(\tau)$. Then, since $\phi$ generates smooth trajectories, we can find a continuous function $r^{0} \in \mathcal{R}_{z}^{\tau}$ such that

$$
r^{0}(\tau)=z \quad \text { and } \quad r^{0}(t) \in \phi(t-s, s, r(s)), \quad \forall t \geqslant s \geqslant \tau .
$$

Notice by Theorem 2.18 that $A$ is invariant under $\phi$, therefore

$$
r^{0}(t) \in \phi(t-\tau, \tau, z) \subset A(t), \quad \forall t \geqslant \tau .
$$

Also, we have

$$
z \in A(\tau) \subset \phi(1, \tau-1, A(\tau-1))
$$

and then there exit a $z_{1} \in A(\tau-1)$ and a family $\mathcal{R}_{z_{1}}^{\tau-1}$ such that

$$
z \in \phi\left(1, \tau-1, z_{1}\right)=\bigcup_{r^{1} \in \mathcal{R}_{z_{1}}^{\tau-1}} r^{1}(\tau) .
$$

Therefore, there exists an $r^{1} \in \mathcal{R}_{z_{1}}^{\tau-1}$ such that

$$
r^{1}(\tau)=z=r^{0}(\tau), r^{1}(\tau-1)=z_{1} \text { and } r^{1}(t) \in \phi\left(t-s, s, r^{1}(s)\right), \forall \tau>t \geqslant s \geqslant \tau-1 .
$$

Continue this process and we obtain a sequence $\left\{r^{n}\right\}_{n=0}^{\infty}$ of continuous functions satisfying

$$
r^{n+1}(\tau-n)=r^{n}(\tau-n) \text { and } r^{n}(t) \in \phi\left(t-s, s, r^{n}(s)\right), \forall \tau-(n-1)>t \geqslant s \geqslant \tau-n .
$$

Now let us define $\xi: \mathbb{R} \rightarrow X$ by

$$
\xi(t)= \begin{cases}r^{0}(t), \quad t \geqslant \tau \\ r^{n}(t), \quad t \in[\tau-n, \tau-n+1) \text { for } n=1,2, \cdots .\end{cases}
$$

Then by the strictness of $\phi$ it is readily verified that the mapping $\xi$ defined by (2.9) is a complete trajectory with $\xi(\tau)=z$. Besides, since this construction of $\xi$ guarantees $\xi \subset A, \xi$ is backwards bounded. The proof is complete.

Proposition 2.25. Let $\phi$ be an $m$-NDS. Suppose for each $x \in X$ and $\tau \in \mathbb{R}$ there exists a family

$$
\tilde{\mathcal{R}}_{x}^{\tau}:=\{r \in C([\tau, \infty) ; X): r(\tau)=x\}
$$

such that

(H1) $\tilde{\mathcal{R}}_{x}^{\tau} \neq \emptyset, \forall \tau \in \mathbb{R}, x \in X$;

(H2) for each $r \in \tilde{\mathcal{R}}_{x}^{\tau}, \tilde{r}:=\left.r(t)\right|_{t \in[\tau+s, \infty)}$ belongs to $\tilde{\mathcal{R}}_{r(\tau+s)}^{\tau+s}, \forall s \geqslant 0$.

If

$$
\phi(t, \tau, x)=\bigcup_{r \in \tilde{\mathcal{R}}_{x}^{\tau}} r(\tau+t), \quad \forall t \geqslant 0,
$$

then $\phi$ generates smooth trajectories satisfying Definition 2.23.

Proof. It suffices to show the family $\tilde{\mathcal{R}}_{x}^{\tau}$ with $(H 1)$ and (H2) is a class satisfying (2.8), that is, to prove $r(t) \in \phi(t-s, s, r(s))$ for each $t \geqslant s \geqslant \tau$ and $r \in \tilde{\mathcal{R}}_{x}^{\tau}$. By (2.10), we only need to find out a $\xi \in \tilde{\mathcal{R}}_{r(s)}^{s}$ such that $\xi(t)=r(t)$. Condition $(H 2)$ indicates that this $\xi$ can be taken as the interception of $r$, that is, take $\xi=\left.r(t)\right|_{t \in[s, \infty)}$. The proof is complete.

Remark 2.26. The structure of attractors in terms of complete trajectories for multi-valued dynamical systems was also studied by $[20,9]$ for uniform attractors and $[14,8]$ for multi-valued semigroups. 


\section{Regularity of pullback attractors for m-NDS}

\subsection{Bi-spatial pullback attractors for strongly dispersive $m$-NDS}

Many m-NDS have a smoothing property that they admit a pullback absorbing set $B$ more regular than the initial data. In this part we study the so-called bi-spatial pullback attractors for smoothing m-NDS.

In the following we let $\left(X,\|\cdot\|_{X}\right)$ and $\left(Y,\|\cdot\|_{Y}\right)$ be two separable Banach spaces with $Y \hookrightarrow X$ continuously except otherwise stated. We first make some elementary definitions.

Definition 3.1. Suppose $\phi$ is an m-NDS in $X$, then $\phi$ is called $Y$-dispersive if $\phi$ has a pullback absorbing set $B$ which is a bounded non-autonomous set in $Y$.

Definition 3.2. We say $K \in \mathcal{D}_{Y}$ is $(X, Y)$-pullback attracting if for every $D \in \mathcal{B}(X)$ we have

$$
\lim _{t \rightarrow \infty} \operatorname{dist}_{Y}\left(\phi\left(t, \theta_{-t} \tau, D\right), K(\tau)\right)=0, \quad \forall \tau \in \mathbb{R} .
$$

Definition 3.3. A mapping $K \in \mathcal{D}_{Y}$ is called an $(X, Y)$-pullback attractor for the m-NDS $\phi$ if

1. $K(\tau)$ is compact in $Y$ for any $\tau \in \mathbb{R}$;

2. $K$ is pullback $(X, Y)$-attracting;

3. $K$ is negatively invariant.

Remark 3.4. Clearly, since $\mathcal{D}_{Y} \subset \mathcal{D}_{X}$, if an m-NDS $\phi$ has pullback $(X, Y)$-attractor $K$ and pullback $(X, X)$-attractor $A$, then $K=A$.

Definition 3.5. An m-NDS $\phi$ in $X$ is called $(X, Y)$-pullback asymptotically compact if for every $D \in \mathcal{B}(X), t_{n} \rightarrow \infty$ and $\tau \in \mathbb{R}$, the sequence $\left\{y_{n}\right\}$ with $y_{n} \in \phi\left(t_{n}, \theta_{-t_{n}} \tau, D\right)$ is precompact in $Y$.

The following result indicates under what conditions the pullback attractor $A$ for a smoothing m-NDS $\phi$ can be bi-spatial.

Proposition 3.6. Let $\phi$ be a $Y$-dispersive $m$-NDS in $X$ with a backwards bounded pullback attractor $A$ in $X$, then $A$ is the $(X, Y)$-pullback attractor of $\phi$ if $\phi$ is $(X, Y)$-pullback asymptotically compact.

Proof. The (negative) invariance follows since $A$ is a pullback attractor for $\phi$. Now let us first prove the $Y$-compactness of $A$. Suppose $\left\{x_{n}\right\}_{n=1}^{\infty} \subset A(\tau), \forall \tau \in \mathbb{R}$, then we should prove $\left\{x_{n}\right\}$ is precompact in $Y$. By the negative invariance and backwards bounded of $A$ we find that, for any sequence $0<t_{k} \rightarrow \infty$,

$$
x_{n} \in \bigcap_{k \in \mathbb{N}} \phi\left(t_{k}, \tau-t_{k}, A\left(\tau-t_{k}\right)\right) \subset \bigcap_{k \in \mathbb{N}} \phi\left(t_{k}, \tau-t_{k}, D\right) \quad \forall n \in \mathbb{N},
$$

for some $D \in \mathcal{B}(X)$. Therefore,

$$
x_{n} \in \phi\left(t_{n}, \tau-t_{n}, D\right) \quad \forall n \in \mathbb{N},
$$

and by the $(X, Y)$-pullback asymptotic compactness we know $\left\{x_{n}\right\}$ is precompact in $Y$.

We then prove the $(X, Y)$-pullback attracting property of $A$ by contradiction. If not, then there exist a $D \in \mathcal{B}(X)$ and a $\delta>0$ such that, for some $\tau \in \mathbb{R}$ and $t_{n} \rightarrow \infty$,

$$
\operatorname{dist}_{Y}\left(\phi\left(t_{n}, \tau-t_{n}, D\right), A(\tau)\right) \geqslant \delta, \quad \forall n \in \mathbb{N} .
$$

By the pullback attraction in $X$ of $A$ we find that there exist a sequence $\left\{x_{n}\right\}$ with $x_{n} \in \phi\left(t_{n}, \tau-t_{n}, D\right)$ such that

$$
\operatorname{dist}_{X}\left(x_{n}, A(\tau)\right) \rightarrow 0, \quad \text { as } n \rightarrow \infty \text {. }
$$


Therefore, by the compactness of $A(\tau)$ we can find an $a \in A(\tau)$ such that, up to a subsequence,

$$
x_{n} \stackrel{X}{\rightarrow} a,
$$

which along with the $(X, Y)$-pullback asymptotic compactness of $A$ implies, up to a subsequence,

$$
x_{n} \stackrel{Y}{\rightarrow} a,
$$

a contradiction. The proof is complete.

Proposition 3.7. Let $\phi$ be a $Y$-dispersive $m$-NDS in $X$. Then if $\phi$ has an $(X, Y)$-pullback attractor $A, \phi$ is $(X, Y)$-pullback asymptotically compact.

Proof. For any $D \in \mathcal{B}(X), t_{n} \rightarrow \infty$ and $\tau \in \mathbb{R}$, we let $\left\{y_{n}\right\}$ be a sequence with $y_{n} \in \phi\left(t_{n}, \theta_{-t_{n}} \tau, D\right)$. By the pullback attraction in $Y$ we have

$$
\operatorname{dist}_{Y}\left(y_{n}, A(\tau)\right) \leqslant \operatorname{dist}_{Y}\left(\phi\left(t_{n}, \theta_{-t_{n}} \tau, D\right), A(\tau)\right) \rightarrow 0 \quad \text { as } n \rightarrow \infty .
$$

Then since $A(\tau)$ is compact in $Y,\left\{y_{n}\right\}$ is precompact in $Y$ as desired.

Remark 3.8. Proposition 3.7 also implies for single-spatial cases (i.e. when $X=Y$ ) that the pullback asymptotic compactness is a necessary condition for the existence of a pullback attractor.

Theorem 3.9. Let $\phi$ be a $Y$-dispersive $m$-NDS in $X$. Suppose $\phi$ has a backwards bounded pullback attractor $A$. Then $A$ is the $(X, Y)$-pullback attractor for $\phi$ if and only if $\phi$ is $(X, Y)$-pullback asymptotically compact.

Proof. This is a direct corollary of the previous two propositions.

Remark 3.10. This theorem indicates that the strong compactness and attracting ability of the attractor, once the attractor exists, are only determined by the dynamical compactness of the system.

Other dynamical compactness of an m-NDS

In this part we present several equivalent dynamical compactnesses which help understand the dynamical property of the m-NDS.

Definition 3.11. An m-NDS $\phi$ is called pullback $\omega$-limit compact if for every $D \in \mathcal{B}(X)$ and $\tau \in \mathbb{R}$,

$$
\lim _{t \rightarrow \infty} \kappa\left(\bigcup_{s \geqslant t} \phi(s, \tau-s, D)\right)=0
$$

where $\kappa$ denotes the Kuratowski measure of noncompactness of sets defined as

$$
\kappa(B)=\inf \{\delta: B \text { has a finite cover by balls of } X \text { of diameter less than } \delta\}, \forall B \subset X .
$$

Definition 3.12. An m-NDS $\phi$ is called pullback flattening if for every $D \in \mathcal{B}(X), \epsilon>0$ and $\tau \in \mathbb{R}$ there exist $T>0$, a finite-dimensional subspace $E$ of $X$ and a mapping $P_{E}: X \rightarrow E$ such that

$$
P_{E}\left(\bigcup_{t \geqslant T} \phi(t, \tau-t, D)\right) \in \mathcal{B}(X)
$$

and

$$
\left\|\left(I-P_{E}\right)\left(\bigcup_{t \geqslant T} \phi(t, \tau-t, D)\right)\right\|_{X}<\epsilon .
$$


Remark 3.13. The pullback flattening property was first introduced by Zhong et al. [22] under the terminology of Condition $\mathrm{C}$ and developed by Kloeden and Langa [10].

Lemma 3.14. [6] Let $X$ be a Banach space and $\phi$ an $m-N D S$ in $X$. Then $\phi$ is pullback flattening $\Rightarrow$ $\phi$ is pullback $\omega$-limit compact $\Leftrightarrow \phi$ is pullback asymptotically compact. If, moreover, $X$ is uniformly convex, then $\phi$ is pullback flattening $\Leftrightarrow \phi$ is pullback $\omega$-limit compact.

\subsection{Backwards precompactness of pullback attractors for $m$-NDS}

Observe that, given a backwards bounded pullback attractor $A \in \mathcal{D}_{X}$ for some m-NDS $\phi$ on $X$, by definition we can find a family of bounded sets $B=\{B(\tau)\}_{\tau \in \mathbb{R}} \in \mathcal{B}(X)$ such that

$$
\overline{\bigcup_{s \leqslant \tau} A(s)} \subset B(\tau), \quad \forall \tau \in \mathbb{R} .
$$

Then if $B$ is moreover compact in $X$, then the attractor $A$ is backwards precompact, namely,

$$
\bigcup_{s \leqslant \tau} A(s) \text { is precompact in } X, \forall \tau \in \mathbb{R} \text {. }
$$

Establishing a compact $B$ with the property (3.1) should be the most direct way to check the backwards compactness of $A$. In many cases especially when Sobolev compactness embeddings hold, $B$ is expectable to be even an increasing and compact pullback absorbing set. In the following we develop a study for more general situations.

Definition 3.15. An m-NDS $\phi$ is called backwards semi-uniformly pullback asymptotically compact if for every $D \in \mathcal{B}(X), \tau \in \mathbb{R}$ and $t_{n} \rightarrow \infty$, any sequence $y_{n} \in \cup_{s \leqslant \tau} \phi\left(t_{n}, s-t_{n}, D\right)$ is precompact in $X$.

Definition 3.16. An m-NDS $\phi$ is called backwards semi-uniformly pullback $\omega$-limit compact if for every $D \in \mathcal{B}(X)$ and $\tau \in \mathbb{R}$,

$$
\lim _{T \rightarrow \infty} \kappa\left(\bigcup_{t \geqslant T} \bigcup_{s \leqslant \tau} \phi(t, s-t, D)\right)=0
$$

where $\kappa$ denotes the Kuratowski measure of noncompactness of sets.

Definition 3.17. An m-NDS $\phi$ is called backwards semi-uniformly pullback flattening if for every $D \in \mathcal{B}(X), \tau \in \mathbb{R}$ and $\epsilon>0$, there exist $T=T_{D, \tau, \epsilon}>0$, a finite subspace $E$ of $X$ and a mapping $P_{E}: X \rightarrow E$ such that

$$
\overline{\bigcup_{t \geqslant T} \bigcup_{s \leqslant \tau} P_{E} \phi(t, s-t, D)} \in \mathcal{B}(X)
$$

and

$$
\sup _{s \leqslant \tau}\left\|\left(I-P_{E}\right)\left(\bigcup_{t \geqslant T} \phi(t, s-t, D)\right)\right\|_{X}<\epsilon .
$$

The following result provides sufficient conditions ensuring a backwards bounded pullback attractor to be backwards precompact.

Theorem 3.18. Suppose $\phi$ is an $m-N D S$ on a Banach space $X$. Then

(i) $\phi$ is backwards semi-uniformly pullback flattening

$\Downarrow$

(ii) $\phi$ is backwards semi-uniforly pullback $\omega$-limit compact

$\Downarrow$

(iii) $\phi$ is backwards semi-uniformly pullback asymptotically compact

$\Downarrow$

(iv) if $\phi$ has a backwards bounded pullback attractor $A$, then $A$ is backwards precompact. 
Proof. (i) $\Rightarrow$ (ii): Let $D \in \mathcal{B}(X)$ and $\tau \in \mathbb{R}$. Then for every $\epsilon>0$, we can find a $T>0$ such that

$$
\begin{aligned}
\kappa\left(\bigcup_{t \geqslant T} \bigcup_{s \leqslant \tau} \phi(t, s-t, D)\right) & \leqslant \kappa\left(\bigcup_{t \geqslant T} \bigcup_{s \leqslant \tau} P_{E} \phi(t, s-t, D)\right)+\kappa\left(\bigcup_{t \geqslant T} \bigcup_{s \leqslant \tau}\left(I-P_{E}\right) \phi(t, s-t, D)\right) \\
& \leqslant 0+\kappa(B(0, \epsilon)) \leqslant \epsilon,
\end{aligned}
$$

where $P_{m}: X \rightarrow E$ with $E$ a finite-dimensional subspace of $X$. Therefore (i) implies (ii).

(ii) $\Rightarrow$ (iii): For every $D \in \mathcal{B}(X), \tau \in \mathbb{R}$ and $t_{n} \rightarrow \infty$, we prove any sequence $y_{n} \in \cup_{s \leqslant \tau} \phi\left(t_{n}, s-\right.$ $\left.t_{n}, D\right)$ is precompact. By condition, for each $\epsilon>0$ there is a $T>0$ such that

$$
\kappa\left(\bigcup_{t \geqslant T} \bigcup_{s \leqslant \tau} \phi(t, s-t, D)\right)<\epsilon
$$

therefore, for $N$ large enough so that $t_{n} \geqslant T$ for all $n \geqslant N$,

$$
\kappa\left(\bigcup_{n \geqslant N} y_{n}\right) \leqslant \kappa\left(\bigcup_{n \geqslant N} \bigcup_{s \leqslant \tau} \phi\left(t_{n}, s-t_{n}, D\right)\right) \leqslant \kappa\left(\bigcup_{t \geqslant T} \bigcup_{s \leqslant \tau} \phi(t, s-t, D)\right)<\epsilon .
$$

Thus (ii) implies (iii).

(iii) $\Rightarrow$ (iv): We need prove (3.2) for $A$. Suppose $\left\{x_{n}\right\}_{n=1}^{\infty}$ is arbitrarily a sequence contained in $\cup_{s \leqslant \tau} A(s)$, and we prove it has a Cauchy subsequence. Suppose also $B$ is a bounded non-autonomous set satisfying (3.1) for $A$ (by the backwards boundedness of $A$ ). Let $x_{n} \in A\left(s_{n}\right), s_{n} \leqslant \tau$. Then, for some $t_{n} \rightarrow \infty$, we have

$$
\begin{aligned}
x_{n} \in A\left(s_{n}\right) & \subset \phi\left(t_{n}, s_{n}-t_{n}, A\left(s_{n}-t_{n}\right)\right) \\
& \subset \phi\left(t_{n}, s_{n}-t_{n}, B(\tau)\right) \\
& \subset \bigcup_{s \leqslant \tau} \phi\left(t_{n}, s-t_{n}, B(\tau)\right) .
\end{aligned}
$$

Therefore $x_{n}$ has a Cauchy subsequence since $\phi$ is backwards semi-uniformly pullback asymptotic compact. The proof is complete.

\section{Applications to a reaction-diffusion type system without uniqueness}

\subsection{Preliminary settings}

\subsubsection{Notations}

Let $d$ be a positive integer and $\mathcal{O} \subset \mathbb{R}^{N}$ with $N \in \mathbb{N}$ a bounded open domain with smooth boundary. Suppose it holds in $\mathcal{O}$ the Poincare inequality for some $\lambda>0$

$$
\|\nabla v\|_{L^{2}(\mathcal{O})}^{2} \geqslant \lambda\|v\|_{L^{2}(\mathcal{O})}^{2} .
$$

We often write as $\left(a_{j}\right)$ an element $\left(a_{1}, \cdots, a_{d}\right)$ of $\mathbb{R}^{d}$ for simplicity. Let $\left(a_{j}\right),\left(b_{j}\right) \in \mathbb{R}^{d}$ be arbitrarily given. Define

$$
\begin{aligned}
& \left(a_{j}\right) \cdot\left(b_{j}\right)=\sum_{j=1}^{d} a_{j} b_{j}, \quad\left(a_{j}\right)+\left(b_{j}\right)=\left(a_{j}+b_{j}\right), \quad\left(a_{j}\right)\left(b_{j}\right)=\left(a_{j} b_{j}\right), \\
& k\left(a_{j}\right)=\left(k a_{j}\right), \quad\left(a_{j}\right)+k=\left(a_{j}+k\right), \quad \forall k \in \mathbb{R} .
\end{aligned}
$$

Let $\mathbf{p}=\left(p_{j}\right)$ be arbitrarily a vector in $\mathbb{R}^{d}$ with $p_{j} \geqslant 2$. Denote $\frac{1}{\mathbf{p}}=\left(\frac{1}{p_{j}}\right)$, and

$$
\left|\left(a_{j}\right)\right|^{\mathbf{p}}=\left(\left|a_{j}\right|^{p_{j}}\right) \in \mathbb{R}^{d} .
$$


Then it makes sense to define the space

$$
L^{\mathbf{p}}(\mathcal{O})=L^{p_{1}}(\mathcal{O}) \times \cdots \times L^{p_{d}}(\mathcal{O})
$$

endowed with the norm

$$
\|u\|_{\mathbf{p}}^{\mathbf{p}}:=\|u\|_{L^{\mathbf{p}(\mathcal{O})}}^{\mathbf{p}}=\sum_{j=1}^{d}\left\|u^{j}\right\|_{L^{p_{j}(\mathcal{O})}}^{p_{j}}
$$

Particularly, we write $H=\left(L^{2}(\mathcal{O})\right)^{d}$ and $V=\left(H_{0}^{1}(\mathcal{O})\right)^{d}$ endowed respectively with the norms

$$
\|u\|:=\|u\|_{H}=\left(\sum_{j=1}^{d}\left\|u^{j}\right\|_{L^{2}(\mathcal{O})}^{2}\right)^{1 / 2}, \quad\|u\|_{V}=\left(\sum_{j=1}^{d}\left\|u^{j}\right\|_{H_{0}^{1}(\mathcal{O})}^{2}\right)^{1 / 2} .
$$

Hereafter throughout this paper, $\mathbf{p}=\left(p_{j}\right)$ is fixed as the vector introduced by the assumptions (4.4), (4.5) on $f$.

\subsubsection{Generating an $m-N D S$}

Consider the following vector-valued equations

$$
\left\{\begin{array}{l}
\frac{\mathrm{d} u}{\mathrm{~d} t}-a \triangle u+f(t, u)=g(x, t) \\
\left.u\right|_{\partial \mathcal{O}}=0 \\
\left.u\right|_{t=\tau}=u_{0}
\end{array}\right.
$$

where $(t, x) \in(\tau, \infty) \times \mathcal{O}, u=\left(u^{1}(t, x), \cdots, u^{d}(t, x)\right):(\tau, \infty) \times \mathcal{O} \rightarrow \mathbb{R}^{d}, a$ is a real $d \times d$ matrix with a positive symmetric part $\frac{a+a^{t}}{2} \geqslant \beta I$ for some $\beta>0 . f=\left(f^{1}(t, u), \cdots, f^{d}(t, u)\right) \in C\left(\mathbb{R} \times \mathbb{R}^{d} ; \mathbb{R}^{d}\right)$ satisfies

$$
\begin{aligned}
& \sum_{j=1}^{d}\left|f^{j}(t, u)\right|^{\frac{p_{j}}{p_{j}-1}} \leqslant c_{1}\left(1+\sum_{j=1}^{d}\left|u^{j}\right|^{p_{j}}\right), \\
& \sum_{j=1}^{d} f^{j}(t, u) u^{j} \geqslant \alpha_{1} \sum_{j=1}^{d}\left|u^{j}\right|^{p_{j}}-c_{2}, \\
& f(t, 0)=0, \quad \forall t \in \mathbb{R},
\end{aligned}
$$

where $p_{j} \geqslant 2$ and $\alpha_{1}, c_{1}, c_{2}$ are all positive constants. We also assume that there exists an $M>0$ such that $f(t, u)$ is continuously differentiable w.r.t. $u$ for all $|u|>M$, and

$$
\frac{\mathrm{d} f(t, u)}{\mathrm{d} u} w \cdot w \geqslant-c_{3}(t)|w|^{2}, \text { for all }|u|>M, w \in \mathbb{R}^{d},
$$

where $c_{3}(t) \in L_{l o c}^{1}\left(\mathbb{R} ; \mathbb{R}^{+}\right)$. For the external force $g(x, t)$ we suppose $g \in L_{l o c}^{2}(\mathbb{R} ; H)$ with a backwards translation bounded property

$$
\sup _{\tau<0} \int_{\tau-1}^{\tau}\|g(s)\|^{2} \mathrm{~d} s<\infty .
$$

The following proposition indicates that condition (4.8) is between translation bounded condition and tempered condition.

Proposition 4.1. Suppose $g \in L_{\text {loc }}^{2}(\mathbb{R} ; H)$. Then condition (4.8) is equivalent to each of the following: 
(G1)

$$
\sup _{\tau<T} \int_{\tau-1}^{\tau}\|g(s)\|^{2} \mathrm{~d} s<\infty, \quad \forall T \in \mathbb{R}
$$

$$
\sup _{\tau<T} \int_{-\infty}^{\tau} e^{\gamma(s-\tau)}\|g(s)\|^{2} \mathrm{~d} s<\infty, \quad \forall \gamma>0, T \in \mathbb{R}
$$

$$
\limsup _{\tau \rightarrow-\infty} \int_{-\infty}^{\tau} e^{\gamma(s-\tau)}\|g(s)\|^{2} \mathrm{~d} s<\infty, \quad \forall \gamma>0 .
$$

Proof. (4.8) $\Rightarrow(G 1)$. It suffices to notice that, since $g \in L_{l o c}^{2}(\mathbb{R} ; H), \sup _{0<\tau<T} \int_{\tau-1}^{\tau}\|g(s)\|^{2} \mathrm{~d} s<\infty$, and thereby for each $T>0$ we have

$$
\sup _{\tau<T} \int_{\tau-1}^{\tau}\|g(s)\|^{2} \mathrm{~d} s \leqslant \sup _{\tau<0} \int_{\tau-1}^{\tau}\|g(s)\|^{2} \mathrm{~d} s+\sup _{0<\tau<T} \int_{\tau-1}^{\tau}\|g(s)\|^{2} \mathrm{~d} s<\infty .
$$

$(G 1) \Rightarrow(G 2)$. For any $\tau<T$,

$$
\begin{aligned}
\int_{-\infty}^{\tau} e^{\gamma(s-\tau)}\|g(s)\|^{2} \mathrm{~d} s & =\sum_{n=0}^{\infty} \int_{\tau-n-1}^{\tau-n} e^{\gamma(s-\tau)}\|g(s)\|^{2} \mathrm{~d} s \\
& \leqslant \sum_{n=0}^{\infty} \int_{\tau-n-1}^{\tau-n} e^{-\gamma n}\|g(s)\|^{2} \mathrm{~d} s \\
& \leqslant \sum_{n=0}^{\infty} e^{-\gamma n}\left(\sup _{t<T} \int_{t-1}^{t}\|g(s)\|^{2} \mathrm{~d} s\right)<\infty .
\end{aligned}
$$

$(G 2) \Rightarrow(G 3)$ is trivial. Let us prove $(G 3) \Rightarrow(4.8)$. Suppose the supper limitation is $c \geqslant 0$. Then there is a time $T<0$ such that

$$
\int_{-\infty}^{\tau} e^{\gamma(s-\tau)}\|g(s)\|^{2} \mathrm{~d} s<c+1, \quad \forall \tau \leqslant T
$$

Therefore, for each $\tau \leqslant T$ we have

$$
\int_{\tau-1}^{\tau}\|g(s)\|^{2} \mathrm{~d} s \leqslant e^{\gamma} \int_{\tau-1}^{\tau} e^{\gamma(s-\tau)}\|g(s)\|^{2} \mathrm{~d} s \leqslant e^{\gamma}(c+1) .
$$

Hence

$$
\sup _{\tau<0} \int_{\tau-1}^{\tau}\|g(s)\|^{2} \mathrm{~d} s \leqslant \sup _{\tau<T} \int_{\tau-1}^{\tau}\|g(s)\|^{2} \mathrm{~d} s+\sup _{T<\tau \leqslant 0} \int_{\tau-1}^{\tau}\|g(s)\|^{2} \mathrm{~d} s<\infty .
$$

The proof is complete.

Definition 4.2. The function $u=u(\cdot)$ is called a weak solution of (4.3) if

$$
u \in C([\tau, T] ; H) \cap L^{\mathbf{p}}\left(\tau, T ; L^{\mathbf{P}}(\mathcal{O})\right) \cap L^{2}(\tau, T ; V), \frac{\partial u}{\partial t} \in L^{\mathbf{q}}\left(\tau, T ; L^{\mathbf{q}}(\mathcal{O})\right)+L^{2}\left(\tau, T ; V^{\prime}\right), u(\tau)=u_{0},
$$

and

$$
\int_{\tau}^{T}\left\langle u_{t}, \psi\right\rangle \mathrm{d} t+\int_{\tau}^{T} \int(a \nabla u, \nabla \psi) \mathrm{d} x \mathrm{~d} t+\int_{\tau}^{T} \int(f(t, u), \psi) \mathrm{d} x \mathrm{~d} t=\int_{\tau}^{T} \int(g, \psi) \mathrm{d} x \mathrm{~d} t
$$

for all $\psi \in L^{\mathbf{p}}\left(\tau, T ; L^{\mathbf{p}}(\mathcal{O})\right) \cap L^{2}(\tau, T ; V)$, where $\langle\cdot, \cdot\rangle$ denotes pairing in the space $V^{\prime}+L^{\mathbf{q}}(\mathcal{O})$. 
Now let us associate an m-NDS to the equation (4.3) in order to study its long time behavior. First, it is known $[16,9,5]$ that under the assumptions (4.4) and (4.5) the problem (4.3) has at least one weak solution $u$ for every given $u_{0} \in L^{2}(\mathcal{O})$. Let us define

$$
\phi\left(t, \tau, u_{0}\right)=\left\{u\left(t+\tau, \tau, u_{0}\right)\right\} \in P(H), \quad \forall t \in \mathbb{R}^{+}, \tau \in \mathbb{R}, u_{0} \in H,
$$

where $u\left(t+\tau, \tau, u_{0}\right)$ is a solution of (4.3) at time $\tau+t$ with initial data $u_{0}$ at time $\tau$.

Proposition 4.3. The set-valued mapping $\phi$ defined by (4.9) is a strict $m$-NDS which generates smooth trajectories in the sense of Definition 2.23. Moreover, it is upper semi-continuous in $H$.

Proof. From the Kneser property [16] it follows $\phi\left(t, \tau, u_{0}\right) \in C(H)$. The strictness property follows from [16, Lemma 3] and the upper semi-continuity from Remark 2.5 and [16, Lemma 2]. $\phi$ indeed satisfies Definition 2.23 since for each $u_{0} \in H$ and $\tau \in \mathbb{R}$ we can take $\mathcal{R}_{u_{0}}^{\tau}=\left\{\left.u\left(t+\tau, \tau, u_{0}\right)\right|_{t \geqslant 0}\right\}$, which satisfies (H1) and (H2) in Proposition 2.25.

\subsection{Backwards precompact attractors in $H$}

In this part, we study the attractor in the phase space $H$. We begin with uniform estimates which are elementary to obtain an absorbing set and the attractor. In the sequel we denote by

$$
\gamma=\beta \lambda
$$

the constant determined by the matrix $a$ and the Poincare property (4.1).

\subsubsection{Uniform estimates}

Proposition 4.4. Let $f(t, u) \in C\left((\tau, \infty) \times \mathbb{R}^{d} ; \mathbb{R}^{d}\right)$ be with the properties (4.6) and (4.7). Then for every $u \in H_{0}^{1} \cap H^{2}$ such that $|u|>M$ it holds the following inequality:

$$
\int f(t, u) \cdot \Delta u \mathrm{~d} x \leqslant c_{3}(t)\|\nabla u\|^{2} .
$$

Proof. Let $f(t, u) \in C^{1}\left((\tau, \infty) \times \mathbb{R}^{d} ; \mathbb{R}^{d}\right)$ admit the properties (4.6) and (4.7). Then, since $\left.f(t, u)\right|_{\partial \mathcal{O}}=$ 0 , by integration by parts we obtain

$$
\begin{aligned}
\int f(t, u) \cdot \triangle u \mathrm{~d} x & =\sum_{k=1}^{n} \sum_{i=1}^{d} \int f^{i}(t, u) \frac{\partial^{2} u^{i}}{\partial x_{k}^{2}} \mathrm{~d} x \\
& =\sum_{k=1}^{n} \sum_{i, j=1}^{d} \int-\frac{\partial f^{i}(t, u)}{\partial u^{j}} \frac{\partial u^{j}}{\partial x_{k}} \frac{\partial u^{i}}{\partial x_{k}} \mathrm{~d} x \\
& =\sum_{k=1}^{n} \int-\frac{\partial f(t, u)}{\partial u} \frac{\partial u}{\partial x_{k}} \cdot \frac{\partial u}{\partial x_{k}} \mathrm{~d} x \\
& \leqslant c_{3}(t) \sum_{k=1}^{n} \int\left|\frac{\partial u}{\partial x_{k}}\right|^{2} \mathrm{~d} x=c_{3}(t)\|\nabla u\|^{2} .
\end{aligned}
$$

Since $f(t, u) \in C\left((\tau, \infty) \times \mathbb{R}^{d} ; \mathbb{R}^{d}\right)$ can be approximated by elements of $C^{1}\left((\tau, \infty) \times \mathbb{R}^{d} ; \mathbb{R}^{d}\right)$, the inequality (4.10) is proved by passing to the limit in (4.11).

Lemma 4.5. Suppose (4.4), (4.5) and (4.8) hold. Then the solution u of (4.3) satisfies

$$
\begin{gathered}
\left\|u\left(\tau, \tau-t, u_{0}\right)\right\|^{2}+\alpha_{1} \int_{\tau-t}^{\tau} e^{\gamma(s-\tau)}\|u(s)\|_{\mathbf{p}}^{\mathbf{p}} \mathrm{d} s+\frac{\beta}{2} \int_{\tau-t}^{\tau} e^{\gamma(s-\tau)}\|\nabla u(s)\|^{2} \mathrm{~d} s \\
\leqslant e^{-\gamma t}\left\|u_{0}\right\|^{2}+c \int_{\tau-t}^{\tau} e^{\gamma(s-\tau)}\|g(s, x)\|^{2} \mathrm{~d} s+c, \quad \forall t \geqslant 0, \tau \in \mathbb{R} .
\end{gathered}
$$


Proof. Multiplying (4.3) by $u$ and integrating over $\mathcal{O}$ we obtain

$$
\frac{1}{2} \frac{\mathrm{d}}{\mathrm{d} t}\|u\|^{2}+\int-a \triangle u \cdot u \mathrm{~d} x=-\int f(t, u) \cdot u \mathrm{~d} x+\int g(x, t) \cdot u \mathrm{~d} x .
$$

Since, by (4.5),

$$
-\int f(t, u) \cdot u \mathrm{~d} x \leqslant-\alpha_{1}\|u\|_{\mathbf{p}}^{\mathbf{p}}+c_{2}|\mathcal{O}|,
$$

by Young's inequality, we have

$$
\frac{\mathrm{d}}{\mathrm{d} t}\|u\|^{2}+2 \beta\|\nabla u\|^{2} \leqslant-2 \alpha_{1}\|u\|_{\mathbf{p}}^{\mathbf{p}}+\frac{\beta \lambda}{2}\|u\|^{2}+c\|g(x, t)\|^{2}+c .
$$

Therefore, since $\gamma=\beta \lambda$ and by Poincare's inequality (4.1), we have

$$
\frac{\mathrm{d}}{\mathrm{d} t}\|u\|^{2}+\gamma\|u\|^{2}+\alpha_{1}\|u\|_{\mathbf{p}}^{\mathbf{p}}+\frac{\beta}{2}\|\nabla u\|^{2} \leqslant c\|g(x, t)\|^{2}+c .
$$

By Gronwall's technique we conclude the lemma.

It will be useful to note that, since $e^{\gamma s} \geqslant e^{\gamma(\tau-1)}$ for all $s \in(\tau-1, \tau)$,

$$
\begin{aligned}
& \alpha_{1} e^{-\gamma} \int_{\tau-1}^{\tau}\left\|u\left(s, \tau-t, u_{0}\right)\right\|_{\mathbf{p}}^{\mathbf{p}} \mathrm{d} s+\frac{\beta}{2} e^{-\gamma} \int_{\tau-1}^{\tau}\left\|\nabla u\left(s, \tau-1, u_{0}\right)\right\|^{2} \mathrm{~d} s \\
& \quad \leqslant \alpha_{1} \int_{\tau-1}^{\tau} e^{\gamma(s-\tau)}\left\|u\left(s, \tau-t, u_{0}\right)\right\|_{\mathbf{p}}^{\mathbf{p}} \mathrm{d} s+\frac{\beta}{2} \int_{\tau-1}^{\tau} e^{\gamma(s-\tau)}\|\nabla u(s)\|^{2} \mathrm{~d} s \\
& \leqslant e^{-\gamma t}\left\|u_{0}\right\|^{2}+c \int_{\tau-t}^{\tau} e^{\gamma(s-\tau)}\|g(s, x)\|^{2} \mathrm{~d} s+c, \quad \forall t \geqslant 1 .
\end{aligned}
$$

Lemma 4.6. Suppose (4.4)-(4.8) hold. Then for every $\tau \in \mathbb{R}$ every solution $u$ of (4.3) satisfies

$$
\sup _{\tau-1 \leqslant s \leqslant \tau}\left\|\nabla u\left(s, \tau-t, u_{0}\right)\right\|^{2} \leqslant L C_{3}^{\tau} e^{-\gamma t}\left\|u_{0}\right\|^{2}+L C_{3}^{\tau} \int_{\tau-t}^{\tau} e^{\gamma(\xi-\tau)}\|g(x, \xi)\|^{2} \mathrm{~d} \xi+L C_{3}^{\tau}, \forall t \geqslant 2,
$$

where $L$ is a positive constant and

$$
C_{3}^{\tau}=e^{\int_{-\infty}^{\tau} 2 c_{3}(\varrho) \mathrm{d} \varrho} \geqslant 1 .
$$

Proof. Multiply (4.3) with $-\triangle u$ and then integrate to obtain

$$
\frac{\mathrm{d}}{\mathrm{d} t}\|\nabla u\|^{2}+2 \beta\|\Delta u\|^{2} \leqslant 2 \int f(t, u) \cdot \Delta u \mathrm{~d} x-2 \int g(x, t) \cdot \Delta u \mathrm{~d} x .
$$

Note that the inequality (4.4) implies a positive constant $C_{M}$ such that

$$
|f(t, u)|^{2} \leqslant C_{M} \quad \text { for all } u \in \mathbb{R}^{d} \text { with }|u| \leqslant M .
$$

Therefore, by (4.10) and (4.14) we obtain

$$
\begin{aligned}
2 \int f(t, u) \cdot \triangle u \mathrm{~d} x & =2 \int_{\{x:|u|>M\}} f(t, u) \cdot \Delta u \mathrm{~d} x+2 \int_{\{x:|u| \leqslant M\}} f(t, u) \cdot \Delta u \mathrm{~d} x \\
& \leqslant 2 c_{3}(t)\|\nabla u\|^{2}+\frac{\beta}{2}\|\triangle u\|^{2}+\frac{2 C_{M}|\mathcal{O}|}{\beta} .
\end{aligned}
$$


Thus, since

$$
-2 \int g(x, t) \cdot \triangle u \mathrm{~d} x \leqslant \frac{\beta}{2}\|\triangle u\|^{2}+\frac{2}{\beta}\|g(x, t)\|^{2},
$$

it follows from (4.13) and (4.15) that

$$
\frac{\mathrm{d}}{\mathrm{d} t}\|\nabla u\|^{2}+\left(\gamma-2 c_{3}(t)\right)\|\nabla u\|^{2} \leqslant c\|g(x, t)\|^{2}+c .
$$

Multiply by $e^{\int_{\tau}^{t}\left(\gamma-2 c_{3}(\varrho)\right) \mathrm{d} \varrho}$ and then integrate w.r.t. $t$ over $(s, \tau)$ where $s \in(\tau-1, \tau)$ to obtain

$$
\begin{aligned}
\left\|\nabla u\left(\tau, \tau-t, u_{0}\right)\right\|^{2} \leqslant & e^{\int_{\tau}^{s}\left(\gamma-2 c_{3}(\varrho)\right) \mathrm{d} \varrho}\left\|\nabla u\left(s, \tau-t, u_{0}\right)\right\|^{2} \\
& +c \int_{\tau-1}^{\tau} e^{\int_{\tau}^{\xi}\left(\gamma-2 c_{3}(\varrho)\right) \mathrm{d} \varrho}\|g(x, \xi)\|^{2} \mathrm{~d} \xi+c \int_{\tau-1}^{\tau} e^{\int_{\tau}^{\xi}\left(\gamma-2 c_{3}(\varrho)\right) \mathrm{d} \varrho} \mathrm{d} \xi \\
\leqslant & c C_{3}^{\tau}\left\|\nabla u\left(s, \tau-t, u_{0}\right)\right\|^{2}+c C_{3}^{\tau} \int_{\tau-1}^{\tau} e^{\gamma(\xi-\tau)}\|g(x, \xi)\|^{2} \mathrm{~d} \xi+c C_{3}^{\tau},
\end{aligned}
$$

where

$$
C_{3}^{\tau}:=e^{\int_{-\infty}^{\tau} 2 c_{3}(\varrho) \mathrm{d} \varrho} \in[1, \infty) .
$$

Integrating (4.17) w.r.t. $s$ over $(\tau-1, \tau)$ and by (4.12) we have, for all $t \geqslant 1$,

$$
\left\|\nabla u\left(\tau, \tau-t, u_{0}\right)\right\|^{2} \leqslant c C_{3}^{\tau} e^{-\gamma t}\left\|u_{0}\right\|^{2}+c C_{3}^{\tau} \int_{\tau-t}^{\tau} e^{\gamma(\xi-\tau)}\|g(x, \xi)\|^{2} \mathrm{~d} \xi+c C_{3}^{\tau} .
$$

On the other hand, for any $s \in[\tau-1, \tau]$ and by taking the place of $\tau$ in (4.19) with $s$, we have

$$
\begin{aligned}
\left\|\nabla u\left(s, \tau-t, u_{0}\right)\right\|^{2} & =\left\|\nabla u\left(s, s-(s-\tau+t), u_{0}\right)\right\|^{2} \\
& \leqslant c C_{3}^{s} e^{-\gamma(s-\tau+t)}\left\|u_{0}\right\|^{2}+c C_{3}^{s} \int_{\tau-t}^{s} e^{\gamma(\xi-s)}\|g(x, \xi)\|^{2} \mathrm{~d} \xi+c C_{3}^{s} \\
& \leqslant c C_{3}^{\tau} e^{-\gamma t}\left\|u_{0}\right\|^{2}+c C_{3}^{\tau} \int_{\tau-t}^{\tau} e^{\gamma(\xi-\tau)}\|g(x, \xi)\|^{2} \mathrm{~d} \xi+c C_{3}^{\tau}
\end{aligned}
$$

for all $s \in[\tau-1, \tau]$ and $t \geqslant 2$. By (4.19) and (4.20) we complete the proof.

Corollary 4.7. Suppose (4.4)-(4.8) hold. Then for each $r>0$, there exists a $T_{0}=\max \left\{\frac{2 \ln r}{\gamma}, 2\right\}$ (so that $e^{-\gamma T_{0}} r^{2} \leqslant 1$ ) such that every solution $u$ of (4.3) with $\left\|u_{0}\right\| \leqslant r$ satisfies

$$
\begin{aligned}
\sup _{t \geqslant T_{0}}\left\|\nabla u\left(\tau, \tau-t, u_{0}\right)\right\|^{2} & \leqslant L C_{3}^{\tau} \int_{-\infty}^{\tau} e^{\gamma(\xi-\tau)}\|g(x, \xi)\|^{2} \mathrm{~d} \xi+2 L C_{3}^{\tau} \\
& \leqslant L C_{3}^{\tau}\left(\sup _{s<\tau} \int_{-\infty}^{s} e^{\gamma(\xi-s)}\|g(x, \xi)\|^{2} \mathrm{~d} \xi\right)+2 L C_{3}^{\tau}, \quad \forall \tau \in \mathbb{R} .
\end{aligned}
$$

4.2.2. Increasing absorbing set and pullback attractor in $H$

Theorem 4.8. Suppose (4.4)-(4.8) hold. Then the m-NDS $\phi$ generated by (4.3) has backwards compact pullback attractor $A$ in $H$. Moreover, $A$ is invariant under $\phi$ and has the presentation

$$
\begin{aligned}
A(\tau) & =\omega(K(\tau), \tau), \quad \forall \tau \in \mathbb{R}, \\
& =\{\xi(\tau): \xi \text { is a complete trajectory backwards compact in } H\}, \forall \tau \in \mathbb{R},
\end{aligned}
$$

where

$$
K(\tau)=\left\{u \in V:\|\nabla u\|^{2} \leqslant L C_{3}^{\tau}\left(\sup _{s<\tau} \int_{-\infty}^{s} e^{\gamma(\xi-s)}\|g(x, \xi)\|^{2} \mathrm{~d} \xi\right)+2 L C_{3}^{\tau}\right\}, \quad \forall \tau \in \mathbb{R} .
$$


Proof. Since $\phi$ is a strict m-NDS, by Theorem 2.18 it suffices to prove $\phi$ has a backwards compact pullback attractor. Clearly, by Corollary 4.7 and Proposition 4.1 (G2) we know $K \in \mathcal{D}_{H}$ is an increasing and compact absorbing set of the system in $H$. By Remark 2.16, the condition (2.2) is satisfied. Therefore, since the compactness of absorbing sets implies the pullback asymptotic compactness, by Theorem 2.15 we conclude (4.21). (4.22) follows from Theorem 2.24 and Proposition 4.3. The proof is complete.

\subsection{Compactness in $V$}

In this part, we study the regularity of attractors for the system (4.3). To achieve this, we suppose the external force $g(x, t)$ is such that

$$
\sup _{\tau<T} \int_{\tau-1}^{\tau}\left(\|g(x, s)\|_{2 \mathbf{p}-2}^{2 \mathbf{p}-2}+\|g(x, s)\|^{2}\right) \mathrm{d} s<\infty, \quad \forall T \in \mathbb{R},
$$

and that

$$
\text { the mapping } \eta \mapsto \int_{-\infty}^{\eta}\|g(x, s)\|^{2} \mathrm{~d} s \text { is uniformly continuous in }(-\infty, \tau), \forall \tau \in \mathbb{R} .
$$

Note that condition (4.25) is for the $V$-backwards compactness of the attractor. We first give a sufficient condition of (4.25) since it is not common in the literature.

Proposition 4.9. Let $g \in L_{\text {loc }}^{2}(\mathbb{R} ; H)$. If there is a time $T \in \mathbb{R}$ such that $\sup _{t \leqslant T}\|g(t)\|^{2}<\infty$, then (4.25) holds.

Proof. We prove by contradiction. If (4.25) does not hold for some $\tau \in \mathbb{R}$, then there exists a $\delta>0$ and for each $n \in \mathbb{N}$ there are $\eta_{1}^{n}, \eta_{2}^{n} \in(-\infty, \tau]$ such that

$$
0<\eta_{2}^{n}-\eta_{1}^{n}<1 / n \quad \text { and } \int_{\eta_{1}^{n}}^{\eta_{2}^{n}}\|g(s)\|^{2} \mathrm{~d} s \geqslant \delta, \quad \forall n \in \mathbb{N} .
$$

Case 1. If $\left\{\eta_{2}^{n}\right\}_{n \in \mathbb{N}}$ is bounded, then there exists an $r \in(-\infty, \tau]$ such that, up to a subsequence, $\eta_{j}^{n} \rightarrow r, j=1,2$. Hence,

$$
\int_{\eta_{1}^{n}}^{\eta_{2}^{n}}\|g(s)\|^{2} \mathrm{~d} s \leqslant \int_{\eta_{1}^{n}}^{r}\|g(s)\|^{2} \mathrm{~d} s+\int_{r}^{\eta_{2}^{n}}\|g(s)\|^{2} \mathrm{~d} s \rightarrow 0, \quad \text { as } n \rightarrow \infty
$$

which contradicts (4.26).

Case 2. If $\left\{\eta_{2}^{n}\right\}_{n \in \mathbb{N}}$ is unbounded, then without loss of generality we have $\sup _{n \in \mathbb{N}} \eta_{2}^{n} \leqslant T$. Then

$$
\int_{\eta_{1}^{n}}^{\eta_{2}^{n}}\|g(s)\|^{2} \mathrm{~d} s \leqslant\left|\eta_{1}^{n}-\eta_{2}^{n}\right| \sup _{s \leqslant T}\|g(s)\|^{2} \leqslant \frac{\sup _{s \leqslant T}\|g(s)\|^{2}}{n} \rightarrow 0, \quad \text { as } n \rightarrow \infty,
$$

which contradicts (4.26) as well. Therefore we have the proposition.

For simplicity we further let $a$ be a diagonal matrix with $\beta=\min \left\{a_{11}, \cdots, a_{d d}\right\} \geqslant 0$. We also assume positive constants $\alpha_{2}$ and $\beta_{i}$ such that

$$
\begin{aligned}
& \sum_{j=1}^{d} f^{j}(t, u) u^{j}\left|u^{j}\right|^{p_{j}-2} \geqslant \alpha_{2} \sum_{j=1}^{d}\left|u^{j}\right|^{2 p_{j}-2}-\beta_{2}, \\
& \sum_{j=1}^{d}\left|f^{j}(t, u)\right|^{2} \leqslant \alpha_{3} \sum_{j=1}^{d}\left|u^{j}\right|^{2 p_{j}-2}+\beta_{3}, \\
& \sum_{j=1}^{d} f^{j}(t, u) u^{j}\left|u^{j}\right|^{2 p_{j}-4} \geqslant-\beta_{1} .
\end{aligned}
$$


Remark 4.10. Assumptions (4.27)-(4.29) are implied by conditions (4.4) and (4.5) when $d=1$. However, in vector-valued case when $d>1$, they should be listed independently.

\subsubsection{Complimentary uniform estimates}

Lemma 4.11. Let (4.4), (4.5) and (4.27) hold. Then for every $\tau \in \mathbb{R}$ the solution $u$ of (4.3) satisfies

$$
\begin{gathered}
\left\|u\left(\tau, \tau-t, u_{0}\right)\right\|_{\mathbf{p}}^{\mathbf{p}}+\frac{\alpha_{2}}{2} \int_{\tau-1}^{\tau} \int_{s}^{\tau} e^{\gamma(\xi-\tau)}\left\|u\left(\xi, \tau-t, u_{0}\right)\right\|_{2 \mathbf{p}-2}^{2 \mathbf{p}-2} \mathrm{~d} \xi \mathrm{d} s \\
\leqslant c e^{-\gamma t}\left\|u_{0}\right\|^{2}+c \int_{\tau-t}^{\tau} e^{\gamma(s-\tau)}\|g(x, s)\|^{2} \mathrm{~d} s+c, \quad \forall t \geqslant 1 .
\end{gathered}
$$

Proof. Observe that

$$
\nabla\left(|u|^{\mathbf{p}-2} u\right)=(\mathbf{p}-1) \nabla u|u|^{\mathbf{p}-2} .
$$

Then by multiplying (4.3) with $|u|^{\mathbf{p}-2} u$ and then integrating we obtain, by (4.27),

$$
\begin{aligned}
& \frac{\mathrm{d}}{\mathrm{d} t} \int \sum_{j=1}^{d} \frac{1}{p_{j}}|u|^{p_{j}} \mathrm{~d} x+\beta \int \sum_{j=1}^{d}\left(p_{j}-1\right)\left|u^{j}\right|^{p_{j}-2}\left|\nabla u^{j}\right|^{2} \mathrm{~d} x \\
& \quad \leqslant-\int f(t, u) \cdot\left(|u|^{\mathbf{p}-2} u\right) \mathrm{d} x+\int g(x, t) \cdot\left(|u|^{\mathbf{p}-2} u\right) \mathrm{d} x \\
& \quad \leqslant-\frac{\alpha_{2}}{2}\|u\|_{2 \mathbf{p}-2}^{2 \mathbf{p}-2}+\beta_{2}|\mathcal{O}|+\frac{1}{\alpha_{2}}\|g(x, t)\|^{2} .
\end{aligned}
$$

Therefore,

$$
\frac{\mathrm{d}}{\mathrm{d} t}\|u\|_{\tilde{\mathbf{p}}}^{\tilde{\mathbf{p}}}+\frac{\alpha_{2}}{2}\|u\|_{2 \mathbf{p}-2}^{2 \mathbf{p}-2} \leqslant c\|g(x, t)\|^{2}+c,
$$

where $\|u\|_{\tilde{\mathbf{p}}}^{\tilde{\mathbf{p}}}$ is an equivalent norm to $\|u\|_{\mathbf{p}}^{\mathbf{p}}$ defined by

$$
\|u\|_{\tilde{\mathbf{p}}}^{\tilde{\tilde{\mathbf{p}}}}=\int \sum_{j=1}^{d} \frac{1}{p_{j}}|u|^{p_{j}} \mathrm{~d} x .
$$

Now multiply (4.32) by $e^{\gamma t}$ and then integrate over $(s, \tau)$ with $s \in(\tau-1, \tau)$ to obtain

$$
\begin{aligned}
& \left\|u\left(\tau, \tau-t, u_{0}\right)\right\|_{\tilde{\mathbf{p}}}^{\tilde{\mathbf{p}}}+\frac{\alpha_{2}}{2} \int_{s}^{\tau} e^{\gamma(\xi-\tau)}\left\|u\left(\xi, \tau-t, u_{0}\right)\right\|_{2 \mathbf{p}-2}^{2 \mathbf{p}-2} \mathrm{~d} \xi \\
& \quad \leqslant c e^{\gamma(s-\tau)}\left\|u\left(s, \tau-t, u_{0}\right)\right\|_{\mathbf{p}}^{\mathbf{p}}+c \int_{\tau-1}^{\tau} e^{\gamma(\xi-\tau)}\|g(x, \xi)\|^{2} \mathrm{~d} \xi+c
\end{aligned}
$$

and then integrate in $s$ over $(\tau-1, \tau)$ to have, by (4.12),

$$
\begin{aligned}
& \left\|u\left(\tau, \tau-t, u_{0}\right)\right\|_{\tilde{\mathbf{p}}}^{\tilde{\mathbf{p}}}+\frac{\alpha_{2}}{2} \int_{\tau-1}^{\tau} \int_{s}^{\tau} e^{\gamma(\xi-\tau)}\left\|u\left(\xi, \tau-t, u_{0}\right)\right\|_{2 \mathbf{p}-2}^{2 \mathbf{p}-2} \mathrm{~d} \xi \mathrm{d} s \\
& \quad \leqslant c \int_{\tau-1}^{\tau} e^{\gamma(s-\tau)}\left\|u\left(s, \tau-t, u_{0}\right)\right\|_{\mathbf{p}}^{\mathbf{p}} \mathrm{d} s+c \int_{\tau-1}^{\tau} e^{\gamma(\xi-\tau)}\|g(x, \xi)\|^{2} \mathrm{~d} \xi+c \\
& \quad \leqslant c e^{-\gamma t}\left\|u_{0}\right\|^{2}+c \int_{\tau-t}^{\tau} e^{\gamma(s-\tau)}\|g(x, s)\|^{2} \mathrm{~d} s+c, \quad \forall t \geqslant 1,
\end{aligned}
$$

which completes the proof since $\left\|u\left(\tau, \tau-t, u_{0}\right)\right\|_{\tilde{\mathbf{p}}}^{\tilde{\mathbf{p}}}$ is equivalent to $\left\|u\left(\tau, \tau-t, u_{0}\right)\right\|_{\mathbf{p}}^{\mathbf{p}}$. 
Lemma 4.12. Let (4.4), (4.5) and (4.24), (4.29) hold. Then for every $\tau \in \mathbb{R}$ the solution $u$ of (4.3) satisfies

$$
\sup _{\tau-\frac{1}{3}<s<\tau}\left\|u\left(s, \tau-t, u_{0}\right)\right\|_{2 \mathbf{p}-2}^{2 \mathbf{p}-2} \leqslant c e^{-\gamma t}\left\|u_{0}\right\|^{2}+c \int_{-\infty}^{\tau} e^{\gamma(s-\tau)}\left(\|g(x, s)\|_{2 \mathbf{p}-2}^{2 \mathbf{p}-2}+\|g(x, s)\|^{2}\right) \mathrm{d} s+c .
$$

Proof. Let us define

$$
\|u\|_{2 \tilde{\mathbf{p}}-2}^{2 \tilde{\mathbf{p}}-2}=\sum_{j=1}^{d}\left(\frac{1}{2 p_{j}-2} \int\left|u^{j}\right|^{2 p_{j}-2} \mathrm{~d} x\right) .
$$

Then $\|u\|_{2 \tilde{\mathbf{p}}-2}^{2 \tilde{\mathbf{p}}-2}$ is an equivalent norm to $\|u\|_{2 \mathbf{p}-2}^{2 \mathbf{p}-2}$.

Multiplying (4.3) by $|u|^{2 \mathbf{p}-4} u$ and then integrating, by (4.29) and Young's inequality we have

$$
\begin{aligned}
& \frac{\mathrm{d}}{\mathrm{d} t}\|u\|_{2 \tilde{\mathbf{p}}-2}^{2 \tilde{\mathbf{p}}-2}+\beta \int \sum_{j=1}^{d}\left(2 p_{j}-3\right)|u|^{2 p_{j}-4}\left|\nabla u^{j}\right|^{2} \mathrm{~d} x \\
& \quad \leqslant-\int f(t, u) \cdot\left(|u|^{2 \mathbf{p}-4} u\right) \mathrm{d} x+\int g(x, t) \cdot\left(|u|^{2 \mathbf{p}-4} u\right) \mathrm{d} x \\
& \quad \leqslant \beta_{1}|\mathcal{O}|+\left(c\|g(x, t)\|_{2 \mathbf{p}-2}^{2 \mathbf{p}-2}+\gamma\|u\|_{2 \tilde{\mathbf{p}}-2}^{2 \tilde{\mathbf{p}}-2}\right),
\end{aligned}
$$

from which it follows

$$
\frac{\mathrm{d}}{\mathrm{d} t}\|u\|_{2 \tilde{\mathbf{p}}-2}^{2 \tilde{\mathbf{p}}-2}-\gamma\|u\|_{2 \tilde{\mathbf{p}}-2}^{2 \tilde{\mathbf{p}}-2} \leqslant c\|g(x, t)\|_{2 \mathbf{p}-2}^{2 \mathbf{p}-2}+c .
$$

Now temporarily we set

$$
\tau-1<\eta \leqslant \tau-2 / 3 \leqslant \xi \leqslant \tau-1 / 3,
$$

and by this we derive an estimate uniformly in $s \in(\tau-1 / 3, \tau)$. Multiply (4.33) by $e^{-\gamma t}$ and then integrate over $(\xi, s)$ to obtain

$$
\begin{aligned}
\left\|u\left(s, \tau-t, u_{0}\right)\right\|_{2 \tilde{\mathbf{p}}-2}^{2 \tilde{\mathbf{p}}-2} \leqslant & c e^{\gamma(\tau-\xi)}\left\|u\left(\xi, \tau-t, u_{0}\right)\right\|_{2 \mathbf{p}-2}^{2 \mathbf{p}-2} \\
& +c \int_{\tau-1}^{\tau} e^{\gamma(s-\varrho)}\|g(x, t)\|_{2 \mathbf{p}-2}^{2 \mathbf{p}-2} \mathrm{~d} \varrho+c .
\end{aligned}
$$

Integrating (4.34) in $\xi$ over $(\eta, s)$ and by $e^{\gamma(\tau-\varrho)} \leqslant e^{2 \gamma} e^{\gamma(\varrho-\tau)}$ for all $\varrho \in[\tau-1, \tau]$ we obtain

$$
\begin{aligned}
(s-\eta)\left\|u\left(s, \tau-t, u_{0}\right)\right\|_{2 \tilde{\mathbf{p}}-2}^{2 \tilde{}-2} \leqslant & c \int_{\eta}^{\tau} e^{\gamma(\tau-\xi)}\left\|u\left(\xi, \tau-t, u_{0}\right)\right\|_{2 \mathbf{p}-2}^{2 \mathbf{p}-2} \mathrm{~d} \xi \\
& +c \int_{\tau-1}^{\tau} e^{\gamma(\tau-\varrho)}\|g(x, s)\|_{2 \mathbf{p}-2}^{2 \mathbf{p}-2} \mathrm{~d} \varrho+c \\
\leqslant & c \int_{\eta}^{\tau} e^{\gamma(\xi-\tau)}\left\|u\left(\xi, \tau-t, u_{0}\right)\right\|_{2 \mathbf{p}-2}^{2 \mathbf{p}-2} \mathrm{~d} \xi \\
& +c \int_{\tau-1}^{\tau} e^{\gamma(\varrho-\tau)}\|g(x, s)\|_{2 \mathbf{p}-2}^{2 \mathbf{p}-2} \mathrm{~d} \varrho+c .
\end{aligned}
$$

Integrating (4.35) in $\eta$ over $(\tau-1, \tau-2 / 3)$ and by (4.30) we have

$$
\begin{aligned}
\left\|u\left(s, \tau-t, u_{0}\right)\right\|_{2 \tilde{\mathbf{p}}-2}^{2 \tilde{\mathbf{p}}-2} \leqslant & c \int_{\tau-1}^{\tau} \int_{\eta}^{\tau} e^{\gamma(\xi-\tau)}\left\|u\left(\xi, \tau-t, u_{0}\right)\right\|_{2 \mathbf{p}-2}^{2 \mathbf{p}-2} \mathrm{~d} \xi \\
& +c \int_{\tau-1}^{\tau} e^{\gamma(\varrho-\tau)}\|g(x, s)\|_{2 \mathbf{p}-2}^{2 \mathbf{p}-2} \mathrm{~d} \varrho+c \\
\leqslant & c e^{-\gamma t}\left\|u_{0}\right\|^{2}+c \int_{-\infty}^{\tau} e^{\gamma(s-\tau)}\left(\|g(x, s)\|_{2 \mathbf{p}-2}^{2 \mathbf{p}-2}+\|g(x, s)\|^{2}\right) \mathrm{d} s+c
\end{aligned}
$$


uniformly in $s \in(\tau-1 / 3, \tau)$. The proof is complete.

\subsection{2. (H,V)-backwards semi-uniformly pullback flattening property}

Consider the continuous compact operator $-\triangle$ in $L^{2}(\mathcal{O})$. It is known by spectral theory that there exist a sequence $\left\{\lambda_{j}\right\}_{j=1}^{\infty}$ with

$$
0<\lambda_{1} \leqslant \lambda_{2} \leqslant \cdots \rightarrow \infty
$$

and a sequence of vectors $\left\{e_{j}\right\}_{j=1}^{\infty} \subset D(-\triangle)$ such that

$$
-\triangle e_{j}=\lambda_{j} e_{j}, \quad j=1,2, \cdots .
$$

Denote by $V_{m}=\left(E_{m}\right)^{d}, \forall m \in \mathbb{N}$, where $E_{m}=\operatorname{span}\left\{e_{1}, e_{2}, \cdots, e_{m}\right\}$ in $H_{0}^{1}(\mathcal{O})$, and $P_{m}: V \mapsto V_{m}$ the orthogonal projector. Then we have the following lemma.

Lemma 4.13. Let (4.4)-(4.7) and (4.24)-(4.29) hold. Then for every $r>0, \epsilon>0$ and $\tau \in \mathbb{R}$, there exist a $T_{0}=\max \left\{\frac{2 \ln r}{\gamma}, 2\right\}$ and an $M=M_{\epsilon, \tau} \in \mathbb{N}$ such that for every solution $u$ of (4.3) with $\left\|u_{0}\right\|<r$ satisfies

$$
\sup _{s \leqslant \tau}\left\|\left(I-P_{M}\right) u\left(s, s-t, u_{0}\right)\right\|^{2}<\epsilon, \quad \forall t \geqslant T_{0} .
$$

Proof. We denote by $u_{m}^{\perp}=\left(I-P_{m}\right) u, \forall m \in \mathbb{N}$. Multiply (4.3) by $A u_{m}^{\perp}$ and then integrate to get

$$
\begin{aligned}
\frac{1}{2} \frac{\mathrm{d}}{\mathrm{d} t}\left\|\nabla u_{m}^{\perp}\right\|^{2}+\beta\left\|\Delta u_{m}^{\perp}\right\|^{2} & \leqslant \int f(t, u) \cdot \triangle u_{m}^{\perp} \mathrm{d} x-\int g(x, t) \cdot \Delta u_{m}^{\perp} \mathrm{d} x \\
& \leqslant \frac{\beta}{2}\left\|\triangle u_{m}^{\perp}\right\|^{2}+\frac{2}{\beta}\|f(t, u)\|^{2}+\frac{2}{\beta}\|g(x, t)\|^{2},
\end{aligned}
$$

and thereby,

$$
\frac{\mathrm{d}}{\mathrm{d} t}\left\|\nabla u_{m}^{\perp}\right\|^{2}+\gamma_{m}\left\|\nabla u_{m}^{\perp}\right\|^{2} \leqslant c\|f(t, u)\|^{2}+c\|g(x, t)\|^{2},
$$

where we have used the relation $\left\|\triangle u_{m}^{\perp}\right\|^{2} \geqslant \lambda_{m}\left\|\nabla u_{m}^{\perp}\right\|^{2}$, and

$$
\gamma_{m}:=\beta \lambda_{m} \text {. }
$$

Since, by (4.28),

$$
\|f(t, u)\|^{2} \leqslant \alpha_{3}\|u\|_{2 \mathbf{p}-2}^{2 \mathbf{p}-2}+c|\mathcal{O}|,
$$

we integrate (4.37) over $(s, \tau)$ with $s \in(\tau-1 / 3, \tau)$ to obtain

$$
\begin{aligned}
\left\|\nabla u_{m}^{\perp}\left(\tau, \tau-t, u_{0}\right)\right\|^{2} \leqslant & e^{\gamma_{m}(s-\tau)}\left\|\nabla u_{m}^{\perp}\left(s, \tau-t, u_{0}\right)\right\|^{2}+c \int_{\tau-1}^{\tau} e^{\gamma_{m}(\xi-\tau)}\|g(x, \xi)\|^{2} \mathrm{~d} \xi \\
& +c \int_{\tau-\frac{1}{3}}^{\tau} e^{\gamma_{m}(\xi-\tau)}\left\|u\left(\xi, \tau-t, u_{0}\right)\right\|_{2 \mathbf{p}-2}^{2 \mathbf{p}-2} \mathrm{~d} \xi+\frac{c}{\gamma_{m}} .
\end{aligned}
$$

Integrating in $s$ over $(\tau-1 / 3, \tau)$ we have

$$
\begin{aligned}
\left\|\nabla u_{m}^{\perp}\left(\tau, \tau-t, u_{0}\right)\right\|^{2} \leqslant & c \int_{\tau-\frac{1}{3}}^{\tau} e^{\gamma_{m}(s-\tau)}\left\|\nabla u_{m}^{\perp}\left(s, \tau-t, u_{0}\right)\right\|^{2} \mathrm{~d} s+c \int_{\tau-1}^{\tau} e^{\gamma_{m}(s-\tau)}\|g(x, s)\|^{2} \mathrm{~d} s \\
& +c \int_{\tau-\frac{1}{3}}^{\tau} e^{\gamma_{m}(\xi-\tau)}\left\|u\left(\xi, \tau-t, u_{0}\right)\right\|_{2 \mathbf{p}-2}^{2 \mathbf{p}-2} \mathrm{~d} \xi+\frac{c}{\gamma_{m}} \\
\leqslant & \frac{c}{\gamma_{m}}\left(\sup _{\tau-\frac{1}{3}<s<\tau}\left\|\nabla u_{m}^{\perp}\left(s, \tau-t, u_{0}\right)\right\|^{2}+\sup _{\tau-\frac{1}{3}<s<\tau}\left\|u\left(s, \tau-t, u_{0}\right)\right\|_{2 \mathbf{p}-2}^{2 \mathbf{p}-2}\right) \\
& +c \int_{\tau-1}^{\tau} e^{\gamma_{m}(s-\tau)}\|g(x, s)\|^{2} \mathrm{~d} s+\frac{c}{\gamma_{m}},
\end{aligned}
$$


where we have used the relation

$$
\int_{\tau-\frac{1}{3}}^{\tau} e^{\gamma_{m}(s-\tau)} \mathrm{d} s=\frac{1}{\gamma_{m}}-\frac{1}{e^{\gamma_{m} / 3}} \leqslant \frac{1}{\gamma_{m}} .
$$

Therefore, by Lemma 4.6 and by Lemma 4.12 , for all $t \geqslant T_{0}$ we have

$$
\begin{aligned}
\left\|\nabla u_{m}^{\perp}\left(\tau, \tau-t, u_{0}\right)\right\|^{2} \leqslant & \frac{c C_{3}^{\tau}}{\gamma_{m}} \int_{-\infty}^{\tau} e^{\gamma(s-\tau)}\left(\|g(x, s)\|_{2 \mathbf{p}-2}^{2 \mathbf{p}-2}+\|g(x, s)\|^{2}\right) \mathrm{d} s \\
& +\frac{c C_{3}^{\tau}}{\gamma_{m}}+c \int_{\tau-1}^{\tau} e^{\gamma_{m}(s-\tau)}\|g(x, s)\|^{2} \mathrm{~d} s,
\end{aligned}
$$

where $C_{3}^{\tau}$ introduced by (4.18) is an increasing function in $\tau$ taking values in $[1, \infty)$. This implies

$$
\begin{aligned}
\sup _{\eta \leqslant \tau}\left\|\nabla u_{m}^{\perp}\left(s, s-t, u_{0}\right)\right\|^{2} \leqslant & \frac{c C_{3}^{\tau}}{\gamma_{m}}\left(\sup _{\eta \leqslant \tau} \int_{-\infty}^{\eta} e^{\gamma(s-\eta)}\left(\|g(x, s)\|_{2 \mathbf{p}-2}^{2 \mathbf{p}-2}+\|g(x, s)\|^{2}\right) \mathrm{d} s\right) \\
& +\frac{c C_{3}^{\tau}}{\gamma_{m}}+c\left(\sup _{\eta \leqslant \tau} \int_{-\infty}^{\eta} e^{\gamma_{m}(s-\eta)}\|g(x, s)\|^{2} \mathrm{~d} s\right), \quad \forall t \geqslant T_{0} .
\end{aligned}
$$

Recall that

$$
\gamma_{m}=\beta \lambda_{m} \rightarrow \infty \quad \text { as } m \rightarrow \infty .
$$

Hence, by condition (4.24) and Proposition 4.14 below, the right-hand side of (4.38) converges to 0 as $m \rightarrow \infty$ for each fixed $\tau \in \mathbb{R}$. Then the lemma is concluded.

Proposition 4.14. Under conditions (4.8), (4.25) and (4.39) it holds

$$
\lim _{m \rightarrow \infty}\left(\sup _{\eta \leqslant \tau} \int_{-\infty}^{\eta} e^{\gamma_{m}(s-\eta)}\|g(x, s)\|^{2} \mathrm{~d} s\right)=0, \quad \forall \tau \in \mathbb{R} .
$$

Proof. Let $\tau \in \mathbb{R}$ be fixed, and set

$$
F(m):=\sup _{\eta \leqslant \tau} \int_{-\infty}^{\eta} e^{\gamma_{m}(s-\eta)}\|g(x, s)\|^{2} \mathrm{~d} s .
$$

Then By (4.8) and Proposition 4.1 (G2) we know $F(m)<\infty$. Since $F(m)$ is non-negative and decreasing, it has a limit $l \geqslant 0$. Suppose $l>0$, and we shall establish a contradiction.

Since $\eta \rightarrow \int_{-\infty}^{\eta}\|g(s)\|^{2} \mathrm{~d} s$ is uniformly continuous in $(-\infty, \tau)$ by $(4.25)$, there exists a $\delta>0$ such that

$$
\sup _{\eta \leqslant \tau} \int_{\eta-\delta}^{\eta}\|g(s)\|^{2} \mathrm{~d} s<l / 4
$$

and thereby

$$
\sup _{\eta \leqslant \tau} \int_{\eta-\delta}^{\eta} e^{\gamma_{m}(s-\eta)}\|g(s)\|^{2} \mathrm{~d} s \leqslant \sup _{\eta \leqslant \tau} \int_{\eta-\delta}^{\eta}\|g(s)\|^{2} \mathrm{~d} s<l / 4, \quad \forall m \in \mathbb{N} .
$$

On the other hand, from Proposition 4.1 (G2) and $\gamma_{m} \rightarrow \infty$ it follows that

$$
\begin{aligned}
\sup _{\eta \leqslant \tau} \int_{-\infty}^{\eta-\delta} e^{\gamma_{m}(s-\eta)}\|g(s)\|^{2} \mathrm{~d} s & =e^{-\gamma_{m} \delta} \sup _{\eta \leqslant \tau} \int_{-\infty}^{\eta-\delta} e^{\gamma_{m}(s-(\eta-\delta))}\|g(s)\|^{2} \mathrm{~d} s \\
& =e^{-\gamma_{m} \delta} \sup _{\eta \leqslant \tau-\delta} \int_{-\infty}^{\eta} e^{\gamma_{m}(s-\eta)}\|g(s)\|^{2} \mathrm{~d} s \\
& \leqslant e^{-\gamma_{m} \delta} \sup _{\eta \leqslant \tau-\delta} \int_{-\infty}^{\eta} e^{\gamma_{1}(s-\eta)}\|g(s)\|^{2} \mathrm{~d} s \rightarrow 0, \quad \text { as } m \rightarrow \infty
\end{aligned}
$$


Therefore,

$$
\begin{aligned}
\sup _{\eta \leqslant \tau} \int_{-\infty}^{\eta} e^{\gamma_{m}(s-\eta)}\|g(s)\|^{2} \mathrm{~d} s & \leqslant \sup _{\eta \leqslant \tau} \int_{\eta-\delta}^{\eta} e^{\gamma_{m}(s-\eta)}\|g(s)\|^{2} \mathrm{~d} s+\sup _{\eta \leqslant \tau} \int_{-\infty}^{\eta-\delta} e^{\gamma_{m}(s-\eta)}\|g(s)\|^{2} \mathrm{~d} s \\
& \leqslant l / 2 \text { for large } m .
\end{aligned}
$$

This contradicts with $l$ being the limit and thereby $l=0$.

Lemma 4.15. Let (4.4)-(4.7) and (4.24)-(4.29) hold. Then the $m$-NDS generated by (4.3) is (H,V)backwards semi-uniformly pullback flattening, namely, for any $D \in \mathcal{B}(H), \tau \in \mathbb{R}$ and $\epsilon>0$, there exist a $T=T_{D, \tau, \epsilon}>0$, a finite-dimensional subspace $E$ of $V$ and a mapping $P_{E}: V \mapsto E$ such that

$$
\bigcup_{s \leqslant \tau} \bigcup_{t \geqslant T} P_{E} \phi(t, s-t, D) \in \mathcal{B}(V),
$$

and

$$
\sup _{s \leqslant \tau} \sup _{t \geqslant T}\left\|\left(I-P_{E}\right) \phi(t, s-t, D)\right\|<\epsilon .
$$

Proof. First recall that by definition,

$$
\phi\left(t, s-t, u_{0}\right)=u\left(s, s-t, u_{0}\right), \quad \forall t \geqslant 0, s \in \mathbb{R}, u_{0} \in H .
$$

Since for any $D \in \mathcal{B}(X)$ there is an $r>0$ such that $\|D\|<r$, by Lemma 4.13, (4.41) is satisfied by taking $T=T_{0}, E=V_{M}$ and $P_{E}=P_{M}$. To see (4.40), by Corollary 4.7 we have

$$
\sup _{s \leqslant \tau} \sup _{t \geqslant T_{0}}\left\|\nabla u\left(s, s-t, u_{0}\right)\right\|^{2} \leqslant L C_{3}^{\tau} \int_{-\infty}^{\tau} e^{\gamma(\xi-\tau)}\|g(x, \xi)\|^{2} \mathrm{~d} \xi+2 L C_{3}^{\tau}, \quad \forall u_{0} \in D .
$$

Hence $\cup_{s \leqslant \tau} \cup_{t \geqslant T} \phi(t, s-t, D) \in \mathcal{B}(V)$, and then (4.40) follows.

\subsubsection{Main result}

Theorem 4.16. Under conditions (4.4)-(4.7) and (4.24)-(4.29), the m-NDS $\phi$ generated by (4.3) has a pullback attractor $A$ defined by (4.21) and with the following properties:

1. A is backwards precompact in $V$, that is,

$$
\bigcup_{s \leqslant \tau} A(s) \quad \text { is precompact in } V, \quad \forall \tau \in \mathbb{R}
$$

2. A attracts every bounded subset of $H$ in the topology of $V$;

3. $A$ is invariant under $\phi$;

4. A has the characterization

$$
A(\tau)=\{\xi(\tau): \xi \text { is a complete trajectory backwards compact in } H\}, \forall \tau \in \mathbb{R} .
$$

Proof. By Theorem 4.8, the non-autonomous set $A$ is a pullback attractor for $\phi$ in $H$ which is invariant and backwards bounded in $V$. Hence the properties 3 and 4 are clear.

Since Lemma 4.15 implies the $(H, V)$-pullback flattening property of $\phi$, from Proposition 3.6 we see that $A$ is moreover the $(H, V)$-pullback attractor of $\phi$. Hence, the property 2 is clear.

To see property 1 , we note that since $A$ is the $(H, V)$-pullback attractor of $\phi$ and $A$ is backwards bounded in $V, A$ is the backwards bounded attractor of $\phi$ in $V$. Then Theorem 3.18 along with the backwards semi-uniformly pullback flattening property in $V$ presented by Lemma 4.15 implies $A$ is backwards compact in $V$. The proof is complete. 


\subsection{An example: attractors for non-autonomous Fitz-Hugh-Nagumo equations}

Now let us study a specific model as an application of the results Theorem 4.8 and Theorem 4.16. Consider the real-valued Fitz-Hugh-Nagumo equation

$$
\left\{\begin{array}{l}
\frac{\partial u_{1}}{\partial t}-a_{11} \Delta u_{1}+b(t) h\left(u_{1}\right)+u_{2}=g_{1}(x, t), \\
\frac{\partial u_{2}}{\partial t}-a_{22} \Delta u_{2}-\delta(t) u_{1}+\xi(t) u_{2}=g_{2}(x, t), \\
\left.u\right|_{\partial \mathcal{O}}=\left.\left(u_{1}, u_{2}\right)\right|_{\partial \mathcal{O}}=0
\end{array}\right.
$$

where $\mathcal{O} \subset \mathbb{R}^{N}$ is a bounded domain with Poincare inequality (4.1). We let $\beta=\min \left\{a_{11}, a_{22}\right\}>0$, and $h(\cdot): \mathbb{R} \mapsto \mathbb{R}$ satisfy $h(0)=0$ and

$$
\begin{cases}|h(s)| \leqslant c_{1}\left(1+|s|^{r-1}\right), & \forall s \in \mathbb{R}, \\ h(s) s \geqslant-c_{2}+c_{3}|s|^{r}, & \forall s \in \mathbb{R}, \\ h^{\prime}(s) \geqslant-c_{4}, & \forall|s|>M,\end{cases}
$$

for some $r \geqslant 2$ and $c_{i}>0, M>0$. Let also $b, \delta, \xi \in C\left(\mathbb{R}, \mathbb{R}^{+}\right)$all with the property

$$
|b(t)| \leqslant c_{4}, \quad \forall t \geqslant 0,
$$

and $b(t) \geqslant \gamma_{1}>0, \xi(t) \geqslant \gamma_{2}>0$. For these constants we further assume

$$
\frac{c_{4}+1}{2}<\min \left\{\gamma_{1} c_{3}, \gamma_{2}\right\}, \quad \text { when } r=2 .
$$

Then it is readily verified that conditions (4.4)-(4.7) and (4.27)-(4.28) are satisfied for all $r \geqslant 2$, and (4.29) are satisfied for $r=2$. Therefore, if we denote by $\mathbf{p}=(r, 2)$ and the external force $g(x, t)=\left(g_{1}, g_{2}\right)$ is assumed with (4.24) and (4.25), then Theorem 4.8 and Theorem 4.16 imply that, the m-NDS generated by the non-autonomous Fitz-Hugh-Nagumo equation (4.42) admits an invariant and backwards compact pullback attractor $A$ in $H$ for all $r \geqslant 2$, and, moreover, $A$ is an $(H, V)$-pullback attractor which is backwards compact in $V$ and composed of backwards bounded complete trajectories if $r=2$.

\section{Acknowledgements}

The authors would like to thank the referees for their carefully reading the script and helpful comments which lead to a great improvement of this work. Hongyong Cui would like to express his sincere thanks to Prof. José A. Langa for his careful guidance and great hospitality during the joint-training program. H. Cui was supported by State Scholarship Fund, J.A. Langa was partially supported by Junta de Andalucía under Proyecto de Excelencia FQM-1492 and Brazilian-European partnership in Dynamical Systems (BREUDS) from the FP7-IRSES grant of the European Union, and Y. Li was partially supported by the NSFC Grant 11571283.

\section{References}

[1] J. BALL, On the asymptotic behavior of generalized processes, with applications to nonlinear evolution equations, Journal of differential equations, 27 (1978), pp. 224-265.

[2] T. Caraballo, M. J. Garrido-Atienza, B. Schmalfuss, and J. Valero, Non-autonomous and random attractors for delay random semilinear equations without uniqueness, Discrete and Continuous Dynamical Systems, 21 (2008), pp. 415-443. 
[3] A. Carvalho, J. A. Langa, and J. Robinson, Attractors for infinite-dimensional nonautonomous dynamical systems, vol. 182, Springer, 2013.

[4] A. N. Carvalho, J. A. Langa, and J. C. Robinson, Non-autonomous dynamical systems, Discrete and Continuous Dynamical Systems-Series b, 20 (2015), pp. 703-747.

[5] V. V. Chepyzhov And M. I. Vishik, Attractors for equations of mathematical physics, vol. 49, American Mathematical Society Providence, RI, 2002.

[6] M. Coti Zelati And P. Kalita, Minimality properties of set-valued processes and their pullback attractors, SIAM Journal on Mathematical Analysis, 47 (2015), pp. 1530-1561.

[7] H. CUI, Y. LI, AND J. Yin, Existence and upper semicontinuity of bi-spatial pullback attractors for smoothing cocycles, Nonlinear Analysis: Theory, Methods \& Applications, 128 (2015), pp. 303-324.

[8] O. V. Kapustyan, P. O. Kasyanov, and J. Valero, Structure and regularity of the global attractor of a reaction-diffusion equation with non-smooth nonlinear term, Discrete and Continuous Dynamical Systems, 34 (2014), pp. 4155-4182.

[9] O. V. Kapustyan, P. O. Kasyanov, J. Valero, and M. Z. Zgurovsky, Structure of uniform global attractor for general non-autonomous reaction-diffusion system, in Continuous and Distributed Systems, Springer, 2014, pp. 163-180.

[10] P. E. Kloeden and J. A. Langa, Flattening, squeezing and the existence of random attractors, Royal Society of London Proceedings Series A, 463 (2007), pp. 163-181.

[11] P. E. Kloeden and M. Rasmussen, Nonautonomous dynamical systems, no. 176, American Mathematical Soc., 2011.

[12] Y. LI, H. CUI, AND J. LI, Upper semi-continuity and regularity of random attractors on p-times integrable spaces and applications, Nonlinear Analysis: Theory, Methods \& Applications, 109 (2014), pp. $33-44$.

[13] Y. LI, A. GU, AND J. LI, Existence and continuity of bi-spatial random attractors and application to stochastic semilinear Laplacian equations, Journal of Differential Equations, 258 (2015), pp. 504 -534 .

[14] J. Simsen And C. B. Gentile, On attractors for multivalued semigroups defined by generalized semiflows, Set-Valued Analysis, 16 (2008), pp. 105-124.

[15] R. Temam, Infinite dimensional dynamical systems in mechanics and physics, Springer-Verlag, New York, 2nd ed., 1997.

[16] J. VAlero And A. Kapustyan, On the connectedness and asymptotic behaviour of solutions of reaction-diffusion systems, Journal of Mathematical Analysis and Applications, 323 (2006), pp. $614-633$.

[17] B. WANG, Sufficient and necessary criteria for existence of pullback attractors for non-compact random dynamical systems, J. Differential Equations, 253 (2012), pp. 1544-1583.

[18] Y. Wang And P. KLOeden, Pullback attractors of a multi-valued process generated by parabolic differential equations with unbounded delays, Nonlinear Analysis: Theory, Methods \& Applications, 90 (2013), pp. 86-95. 
[19] Y. WANG AND J. WANG, Pullback attractors for multi-valued non-compact random dynamical systems generated by reaction-diffusion equations on an unbounded domain, Journal of Differential Equations, 259 (2015), pp. 728-776.

[20] Y. WANG AND S. Zhou, Kernel sections and uniform attractors of multi-valued semiprocesses, Journal of Differential Equations, 232 (2007), pp. 573-622.

[21] M. Z. Zgurovsky, P. Kasyanov, O. V. Kapustyan, J. Valero, and N. V. Zadoianchuk, Evolution Inclusions and Variation Inequalities for Earth Data Processing III: Long-Time Behavior of Evolution Inclusions Solutions in Earth Data Analysis, vol. 27, Springer Science \& Business Media, 2012.

[22] C. Zhong, M. YAng, And C. Sun, The existence of global attractors for the norm-to-weak continuous semigroup and application to the nonlinear reactiondiffusion equations, Journal of Differential Equations, 223 (2006), pp. $367-399$. 